\title{
ANALYSIS OF LINEAR VARIABLE COEFFICIENT DELAY DIFFERENTIAL-ALGEBRAIC EQUATIONS
}

\author{
PHI $\mathrm{HA}^{\dagger *}$, VOLKER MEHRMANN ${ }^{\dagger \ddagger}$, AND ANDREAS STEINBRECHER ${ }^{\dagger \ddagger}$ \\ June 28, 2013
}

\begin{abstract}
The analysis of general linear variable coefficient delay differential-algebraic systems (DDAEs) is presented. The solvability for DDAEs is investigated and a reformulation procedure to regularize a given DDAE is developed. Based on this regularization procedure existence and uniqueness of solutions and consistency of initial functions is analyzed as well as other structural properties of DDAEs like smoothness requirements. We also present some examples to demonstrate that for the numerical solution of a DDAE, a reformulation of the system before applying numerical methods is essential.
\end{abstract}

Keywords: Differential-algebraic equation, delay differential-algebraic equation, regularization, existence of solutions, uniqueness of solutions, consistency conditions.

AMS Subject Classification: 34A09, 34A12, 65L05, 65H10

1. Introduction. In this paper we study general linear delay differential-algebraic equations (DDAEs) with variable coefficients of the form

$$
E(t) \dot{x}(t)=A(t) x(t)+B(t) \Delta_{\tau} x(t)+f(t),
$$

on the time interval $\mathbb{I}=[0, \infty)$, where $\dot{x}$ denotes the first (time) derivative of the vector valued function $x, \Delta_{\tau}$ denotes the shift (backward) operator, i.e., $\Delta_{\tau} x: t \mapsto x(t-\tau)$, with a single constant delay $\tau>0$. The coefficients are matrix functions $E, A, B: \mathbb{I} \rightarrow \mathbb{C}^{m, n}, f: \mathbb{I} \rightarrow \mathbb{C}^{m}$. Most of the results in this paper can also be extended to multiple delays, but here we only discuss the single delay case.

Note that DDAEs of the form (1.1) include many classes of dynamical systems. In particular, we focus on: differential-algebraic equations (DAEs)

$$
E(t) \dot{x}(t)=A(t) x(t)+f(t),
$$

difference equations

$$
0=A(t) x(t)+B(t) \Delta_{\tau} x(t)+f(t),
$$

and also delay-differential equations

$$
\dot{x}(t)=A(t) x(t)+B(t) \Delta_{\tau} x(t)+f(t) .
$$

Standard DAEs without delay of the form 1.2 are common mathematical models in many application areas, such as multibody systems, electrical circuit simulation, control theory, fluid dynamics, chemical engineering, see e. g. [1, 5, 17, 21, 26] and the references therein. However, in a more realistic way, evolution phenomena arising in physics, biology, chemistry, or engineering should be modeled with hereditary characteristics such as memory and time delays, and thus naturally delay

${ }^{*}$ This author has been supported by Deutsche Forschungsgemeinschaft through Sonderforschungsbereich 910 Control of self-organizing nonlinear systems: Theoretical methods and application concepts

${ }^{\ddagger}$ This author has been supported by European Research Council through Advanced Grant MODSIMCONMP

${ }^{\dagger}$ Institut für Mathematik, MA 4-5, TU Berlin, Straße des 17. Juni 136, D-10623 Berlin, Germany; \{ha,mehrmann,steinbrecher\}@math.tu-berlin.de 
differential-algebraic equations arise. The delay may arise due to the computing time needed for extensive computations [12, due to interconnects for networks [20, 29], in lossless transmission lines [18, 30, or in fluid flow models [19, 24]. In particular, linear delay differential-algebraic equations arise after linearizing general nonlinear delay differential-algebraic equations of the form

$$
F\left(t, x(t), \dot{x}(t), \Delta_{\tau} x(t)\right)=0
$$

around a non-constant trajectory. We demonstrate that by the following example.

EXAMPLE 1.1. The dynamical behavior of a system in fluid mechanics and turbulence modeling is often described by the incompressible Navier-Stokes equation of the form

$$
\begin{aligned}
\frac{\partial u}{\partial t}-\nu \Delta u+\nabla p+(u \cdot \nabla) u & =F, \\
\nabla \cdot u & =0,
\end{aligned}
$$

where $\nu>0$ is the viscosity, $u=u(t, \xi)$ is the velocity field which is a function of the time $t$ and the position $\xi, p$ is the pressure, $F$ is the external force. Recently, there has been an increasing interest in the situation where the trajectories of some fluid particles have a delay $\tau$ to follow the fluid [22, 25]. Furthermore, from the control perspective, it is favorable to control the system by some external force $G=G(t, u(t-\tau, \xi))$ which involves some hereditary characteristics [9, 11]. This leads to the following time-delayed version of the incompressible Navier-Stokes equation

$$
\begin{aligned}
\frac{\partial u}{\partial t}-\nu \Delta u+\nabla p+(u(t-\tau, \xi) \cdot \nabla) u & =F+G(t, u(t-\tau, \xi)), \\
\nabla \cdot u & =0,
\end{aligned}
$$

together with suitable initial and boundary conditions. Then, using linearization around a trajectory and discretizing the space variable by finite difference or finite element methods [14], one obtains a delay differential-algebraic system of the form (1.1).

To achieve uniqueness of solutions, for DDEs of the form (1.4) one typically has to prescribe initial functions of the form

$$
\left.x\right|_{[-\tau, 0]}=\phi:[-\tau, 0] \rightarrow \mathbb{C}^{n} .
$$

However, as for standard DAEs these functions cannot be described arbitrarily but must satisfy certain consistency conditions, and as we will see, certain extra smoothness requirements.

Although DAEs are well studied analytically and numerically, see e. g. [5, 13, 17, 21, the theoretical understanding of DDAEs and the development of appropriate numerical methods is far from complete even for the case of linear systems with constant coefficients. Only few results are available, see e. g., 2, 3, 6, 7, 8, 16, 27.

The main difficulty in the theoretical analysis so far is the lack of a suitable regularity analysis and a suitable reformulation of the problem which allows to investigate structural properties like existence, uniqueness of solutions, consistency and smoothness requirements for an initial function and an inhomogeneity.

On the other hand, from the numerical point of view, directly applying numerical methods such as Runge-Kutta or BDF methods to a given DDAE may fail or provide a wrong solution. However, if we first reformulate a given DDAE to a suitable form, then these methods work fine. This observation is demonstrated in the following example.

EXAmple 1.2. Consider the system

$$
\left[\begin{array}{cc}
0 & 0 \\
1 & -t
\end{array}\right]\left[\begin{array}{l}
\dot{x}_{1}(t) \\
\dot{x}_{2}(t)
\end{array}\right]=\left[\begin{array}{cc}
1 & -(t-1) \\
1 & t
\end{array}\right]\left[\begin{array}{l}
x_{1}(t-\tau) \\
x_{2}(t-\tau)
\end{array}\right]+\left[\begin{array}{c}
0 \\
2-2 t
\end{array}\right]
$$


in the time interval $t \in \mathbb{I}=[0, \infty)$, with delay $\tau=1$ and initial function $\phi(t)=\left[\begin{array}{l}t \\ 1\end{array}\right], t \in[-\tau, 0]$. Despite the fact that this system has a unique solution $x_{e}(t)=\left[\begin{array}{l}t \\ 1\end{array}\right]$, numerical methods such as Runge-Kutta or BDF methods do not work for system (1.6), since the Jacobian of the discretized system of the non-delay part is singular. However, if one first reformulates system (1.6) by shifting forward the first equation and then adding its derivative to the second equation to get

$$
\left[\begin{array}{l}
0 \\
0
\end{array}\right]=\left[\begin{array}{ll}
1 & -t \\
0 & -1
\end{array}\right]\left[\begin{array}{l}
x_{1}(t) \\
x_{2}(t)
\end{array}\right]+\left[\begin{array}{ll}
0 & 0 \\
1 & t
\end{array}\right]\left[\begin{array}{l}
x_{1}(t-\tau) \\
x_{2}(t-\tau)
\end{array}\right]+\left[\begin{array}{c}
0 \\
2-2 t
\end{array}\right]
$$

then these numerical methods successfully work for 1.7 . The numerical integration is demonstrated in Figure 1.1, where we have used the 3 stage Radau IIA method of [15] with step size $h=0.01$ for system (1.7).
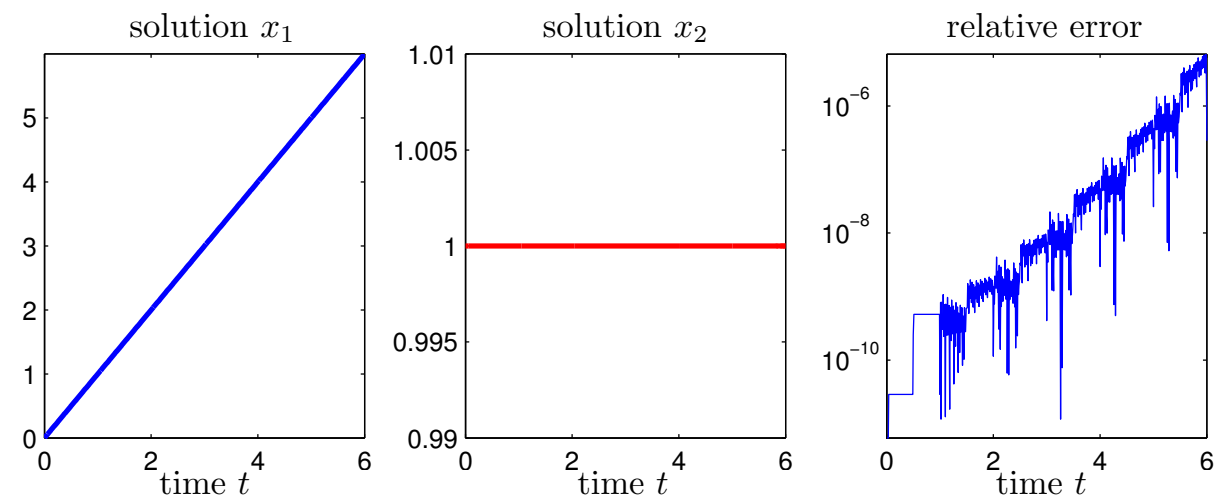

FIG. 1.1. Solution of system 1.7 together with the relative error $\left\|x(t)-x_{e}(t)\right\|_{\infty} /\left\|x_{e}(t)\right\|_{\infty}$

It is the aim of this paper to derive a reformulation as in Example 1.2 for general linear variable coefficient DDAEs by extending the algebraic approach introduced in [21, 28]. The outline of the paper is as follows. In Section 2 we recall some preliminary concepts and some auxiliary lemmas that will be used later. As a preparation step, Section 3 studies two special cases of the DDAE (1.1), the systems $(1.2)$ and (1.3). Then, our main results for the analysis of system (1.1) will be presented in Section 4 . We introduce a reformulation procedure which brings the systems into a form that allows to read off all the constraints that are contained in the system and to analyze theoretical aspects such as solvability, uniqueness, consistency, and the necessary smoothness requirements. We finish with some examples to illustrate our results and to demonstrate the necessity of a reformulation procedure to DDAEs.

2. Notation and preliminaries. In the following we denote by $\mathbb{N}\left(\mathbb{N}_{0}\right)$ the set of natural numbers (including 0 ), by $\mathbb{R}(\mathbb{C})$ the set of real (complex) numbers. By $\mathbb{C}^{m, n}$ we denote the complex matrices of size $m \times n$ and by $I\left(I_{n}\right)$ the identity matrix (of size $n \times n$ ). As usual $x^{(j)}$ is the $j$-th derivative of a function $x: \mathbb{I} \rightarrow \mathbb{C}^{n}$. By $\Delta_{-\tau}$ we denote a shift (forward) operator $\Delta_{-\tau}: M(t) \mapsto M(t+\tau)$. By $\mathbb{I}_{\tau}$ we denote the time interval $[-\tau, \infty)$. By $C^{k}\left(\mathbb{I}, \mathbb{C}^{n}\right)$ we denote the space of $k$-times continuously differentiable functions from $\mathbb{I}$ to $\mathbb{C}^{n}$.

For differential-algebraic equations, one frequently uses the concept of classical solutions, i.e., functions $x: \mathbb{I} \rightarrow \mathbb{C}^{n}$ that are continuously differentiable and satisfy 1.1) with $B=0$ pointwise, see e.g. 4, 5. However, in the theory of delay differential equations, there is no reason why $E(0) \dot{x}(0)$ which arises in (1.1) should be equal to $E(0) \dot{\phi}\left(0^{-}\right)$. For delay differential-algebraic equations, it 
has been observed in [3, 6, 15] that a discontinuity of $\dot{x}$ at $t=0$ may propagate with time, and typically $\dot{x}$ is discontinuous at every point $j \tau, j \in \mathbb{N}_{0}$. To deal with this property of DDAEs, we use the following solution concept.

Definition 2.1. A function $x: \mathbb{I}_{\tau} \rightarrow \mathbb{C}^{n}$ is called a piecewise differentiable solution of (1.1), if it is continuous, piecewise continuously differentiable and satisfies 1.1 at every $t \in \mathbb{I} \backslash \underset{j \in \mathbb{N}_{0}}{\cup}\{j \tau\}$.

Throughout this paper whenever we speak of a solution, we mean a piecewise differentiable solution.

REMARK 2.2. It is possible that if the initial function $\phi$ is discontinuous, then this may lead to solutions that are only piecewise continuous, but here we do not discuss such solutions and assume that the initial function $\phi$ is continuous.

As for standard DAEs one cannot prescribe arbitrary initial conditions, but they have to obey certain consistency conditions.

Definition 2.3. An initial function $\phi$ is called consistent with (1.1) if the associated initial value problem (1.1), 1.5 has at least one solution. System (1.1) is called solvable (resp. regular) if for every consistent initial function $\phi$, the associated initial value problem 1.1), 1.5 has a solution (resp. has a unique solution).

We will make frequent use of the following results, compare Theorems 3.9, 3.25 in [21].

Theorem 2.4. Let $E \in C^{\ell}\left(\mathbb{I}, \mathbb{C}^{m, n}\right), \ell \in \mathbb{N}_{0} \cup\{\infty\}$, with constant $\operatorname{rank} E(t)=r$ for all $t \in \mathbb{I}$. Then there exist pointwise nonsingular functions $U \in C^{\ell}\left(\mathbb{I}, \mathbb{C}^{m, m}\right)$ and $V \in C^{\ell}\left(\mathbb{I}, \mathbb{C}^{n, n}\right)$, such that

$$
U^{H} E V=\left[\begin{array}{cc}
\Sigma & 0 \\
0 & 0
\end{array}\right], \quad \text { or } \quad U^{H} E=\left[\begin{array}{c}
E_{1} \\
0
\end{array}\right]
$$

with pointwise nonsingular $\Sigma \in C^{\ell}\left(\mathbb{I}, \mathbb{C}^{r, r}\right)$, and $E_{1}$ has full row rank $r$.

TheOREm 2.5. Let $\mathbb{I} \subset \mathbb{R}$ be a closed interval and $M \in C\left(\mathbb{I}, \mathbb{C}^{m, n}\right)$. Then there exist open intervals $\mathbb{I}_{j} \subset \mathbb{I}, j \in \mathbb{N}$, with

$$
\overline{\bigcup_{j \in \mathbb{N}} \mathbb{I}_{j}}=\mathbb{I}, \quad \mathbb{I}_{i} \cap \mathbb{I}_{j}=\emptyset \text { for } i \neq j
$$

and integers $r_{j} \in \mathbb{N}_{0}, j \in \mathbb{N}$ such that

$$
\operatorname{rank} M(t)=r_{j} \text { for all } t \in \mathbb{I}_{j} .
$$

For two matrix functions $P \in C\left(\mathbb{I}, \mathbb{C}^{p, n}\right), Q \in C\left(\mathbb{I}, \mathbb{C}^{q, n}\right)$, the pair $(P, Q)$ is said to have no hidden redundancy if

$$
\operatorname{rank}\left[\begin{array}{c}
P(t) \\
Q(t)
\end{array}\right]=\operatorname{rank} P(t)+\operatorname{rank} Q(t)
$$

for all $t \in \mathbb{I}$.

Lemma 2.6. Suppose that for $P \in C\left(\mathbb{I}, \mathbb{C}^{p, n}\right), Q \in C\left(\mathbb{I}, \mathbb{C}^{q, n}\right)$, the pair $(P, Q)$ has no hidden redundancy. Let $U_{1} \in C\left(\mathbb{I}, \mathbb{C}^{p, p}\right), U_{2} \in C\left(\mathbb{I}, \mathbb{C}^{q, q}\right), V \in C\left(\mathbb{I}, \mathbb{C}^{n, n}\right)$ be pointwise invertible functions. Then, the pair of functions $\left(U_{1} P V, U_{2} Q V\right)$ has no hidden redundancy.

Proof. The proof follows from the observation that a pair of matrix functions has no hidden redundancy if and only if the intersection of the two vector spaces spanned by the rows of the two 
matrices contains only the vector 0 .

The following lemma shows how one can remove the hidden redundancy of a pair of matrix-valued functions.

Lemma 2.7. For the pair $(P, Q)$ with $P \in C\left(\mathbb{I}, \mathbb{C}^{p, n}\right), Q \in C\left(\mathbb{I}, \mathbb{C}^{q, n}\right)$, assume that there exist two integers $r_{Q} \leqslant r_{[P ; Q]}$ such that $\operatorname{rank} Q(t)=r_{Q}$ and $\operatorname{rank}\left[\begin{array}{c}P(t) \\ Q(t)\end{array}\right]=r_{[P ; Q]}$ for all $t \in \mathbb{I}$. Then, there exists $\left[\begin{array}{cc}S & 0 \\ Z_{1} & Z_{2}\end{array}\right] \in C\left(\mathbb{I}, \mathbb{C}^{p, p+q}\right)$ that satisfies the following conditions.

i) $\left[\begin{array}{l}S \\ Z_{1}\end{array}\right] \in C\left(\mathbb{I}, \mathbb{C}^{p, p}\right)$ is pointwise nonsingular,

ii) $Z_{1} P+Z_{2} Q=0$,

iii) the function $S P$ has pointwise full row rank, and the pair $(S P, Q)$ has no hidden redundancy.

Proof. Since $Q$ has constant rank on $\mathbb{I}$, one can apply Theorem 2.4 to factorize $Q$, and then partition $P$ conformably to get

$$
\left[\begin{array}{cc}
I_{p} & 0 \\
0 & U_{11}^{H} \\
0 & U_{12}^{H}
\end{array}\right]\left[\begin{array}{c}
P \\
Q
\end{array}\right]\left[\begin{array}{ll}
V_{11} & V_{12}
\end{array}\right]=\left[\begin{array}{cc}
P_{1} & P_{2} \\
\Sigma & 0 \\
0 & 0
\end{array}\right] \begin{gathered}
p \\
r_{Q} \\
q-r_{Q}
\end{gathered}
$$

where $U_{1}=\left[\begin{array}{ll}U_{11} & U_{12}\end{array}\right] \in C\left(\mathbb{I}, \mathbb{C}^{q, q}\right), V_{1}=\left[\begin{array}{ll}V_{11} & V_{12}\end{array}\right] \in C\left(\mathbb{I}, \mathbb{C}^{n, n}\right)$ are pointwise nonsingular functions, and $\Sigma \in C\left(\mathbb{I}, \mathbb{C}^{r_{Q}}, r_{Q}\right)$ is pointwise nonsingular. The sizes of the block rows in 2.1) are $p, r_{Q}, q-r_{Q}$. Moreover, note that in 2.1., $P_{2}$ also has constant rank due to

$$
\operatorname{rank}\left(P_{2}(t)\right)=\operatorname{rank}\left(\left[\begin{array}{cc}
I_{p} & 0 \\
0 & U_{1}^{H}
\end{array}\right]\left[\begin{array}{l}
P \\
Q
\end{array}\right]\left[\begin{array}{ll}
V_{11} & V_{12}
\end{array}\right]\right)-\operatorname{rank}(\Sigma)=r_{[P ; Q]}-r_{Q}
$$

Then, by Theorem 2.4 there exists a pointwise nonsingular function $U_{2}^{H}=\left[\begin{array}{l}S \\ Z_{1}\end{array}\right] \in C\left(\mathbb{I}, \mathbb{C}^{p, p}\right)$ such that

$$
U_{2}^{H} P_{2}=\left[\begin{array}{c}
S \\
Z_{1}
\end{array}\right] P_{2}=\left[\begin{array}{c}
P_{12} \\
0
\end{array}\right]
$$

where $P_{12}$ has pointwise full row rank $r_{[P ; Q]}-r_{Q}$.

Combining 2.1 and 2.2, one obtains

$$
\left[\begin{array}{cc}
S & 0 \\
Z_{1} & 0 \\
0 & U_{11}^{H} \\
0 & U_{12}^{H}
\end{array}\right]\left[\begin{array}{l}
P \\
Q
\end{array}\right]\left[\begin{array}{ll}
V_{11} & V_{12}
\end{array}\right]=\left[\begin{array}{cc}
P_{11} & P_{12} \\
P_{21} & 0 \\
\Sigma & 0 \\
0 & 0
\end{array}\right]
$$

where $P_{12}$ has pointwise full row rank and $\Sigma$ is pointwise nonsingular on $\mathbb{I}$.

By definition, the function pair $\left(\left[\begin{array}{ll}P_{11} & P_{12}\end{array}\right],\left[\begin{array}{ll}\Sigma & 0 \\ 0 & 0\end{array}\right]\right)$ has no hidden redundancy, and due to Lemma 2.6, so does $(S P, Q)$. Since $P_{12}$ has pointwise full row rank, so does $S P=\left[\begin{array}{ll}P_{11} & P_{12}\end{array}\right] V_{1}^{-1}$. Furthermore, setting $Z_{2}:=P_{21} \Sigma^{-1} U_{11}^{H}$ we obtain

$$
Z_{1} P-Z_{2} Q=\left(\left[\begin{array}{ll}
P_{21} & 0
\end{array}\right]-P_{21} \Sigma^{-1}\left[\begin{array}{ll}
\Sigma & 0
\end{array}\right]\right) V_{1}^{-1}=0,
$$

which completes the proof. 
REMARK 2.8. Note that in Lemma 2.7 the matrix-valued function $\left[\begin{array}{c}S \\ Z_{1}\end{array}\right]$ can be chosen to have pointwise orthonormal columns. This orthonormal representation is preferred for stability reasons in numerical computations.

\section{Analysis of differential-algebraic and difference equations. As a} preparation step for the analysis of DDAEs, this section is devoted to two special cases of the DDAE (1.1), the differential-algebraic equation (1.2) and the difference equation (1.3). We recall that the considered time interval is $\mathbb{I}=[0, \infty)$. For notational convenience, in this section we omit the time-variable $t$ in all matrix-valued and vector-valued functions.

3.1. Differential-algebraic equations. With $M:=\left[\begin{array}{ll}E & -A\end{array}\right]$, we can rewrite system 1.2 in the form

$$
M\left[\begin{array}{l}
\dot{x} \\
x
\end{array}\right]=f .
$$

Making use of Theorem 2.5 and restricting ourselves if necessary to subintervals, we may assume that the following assumption holds.

Assumption 3.1. For the pair of matrix valued functions $(E, A)$ of the DAE $(1.2)$, there exist integers $r$, a such that

$$
\operatorname{rank}(E)=r, \operatorname{rank}(M)=r+a \text { for all } t \in \mathbb{I} .
$$

Lemma 3.2. Consider the DAE (1.2) and suppose that Assumption 3.1 holds for the pair $(E, A)$. Then, there exists a pointwise nonsingular function $P_{1} \in C\left(\mathbb{I}, \mathbb{C}^{m, m}\right)$ such that by scaling system (3.1) with $P_{1}$ from the left one obtains a new system in the block upper triangular form

$$
\left[\begin{array}{cc}
M_{11} & M_{12} \\
0 & M_{22} \\
0 & 0
\end{array}\right]\left[\begin{array}{l}
\dot{x} \\
x
\end{array}\right]=\left[\begin{array}{l}
f_{1} \\
f_{2} \\
f_{3}
\end{array}\right] \begin{gathered}
r \\
a \\
v=m-r-a
\end{gathered},
$$

where the functions $M_{11} \in C\left(\mathbb{I}, \mathbb{C}^{r, n}\right), M_{22} \in C\left(\mathbb{I}, \mathbb{C}^{a, n}\right)$ have pointwise full row rank.

Proof. We first compress the block column $E$ of $M$ with a pointwise nonsingular function $P_{E}: \mathbb{I} \rightarrow \mathbb{C}^{m, m}$ via Theorem 2.4 or a smooth QR-decomposition, see [10, yielding

$$
P_{E} M=\left[\begin{array}{cc}
\widetilde{M}_{11} & \widetilde{M}_{12} \\
0 & \widetilde{M}_{22}
\end{array}\right] m-r
$$

such that $\widetilde{M}_{11}$ has full row rank. Continuing, by compressing the second block column from the second block row with a pointwise nonsingular function $P_{A}=\left[\begin{array}{cc}I & 0 \\ 0 & \tilde{P}_{A}\end{array}\right]: \mathbb{I} \rightarrow \mathbb{C}^{m, m}, \tilde{P}_{A} \widetilde{M}_{22}=\left[\begin{array}{c}M_{22} \\ 0\end{array}\right]$ where $M_{22}$ has full row rank $a$. Setting $P_{1}:=P_{A} P_{E}$ we arrive at $(3.3)$.

Again, to be able to apply Lemma 2.7, we assume that the following assumption holds.

Assumption 3.3. For the DAE (1.2) and the equivalent DAE (3.3), the functions $M_{11}, M_{22}$ satisfy the constant rank identity

$$
\operatorname{rank}\left(\left[\begin{array}{l}
M_{11} \\
M_{22}
\end{array}\right]\right)=\widehat{m}, \text { for all } t \in \mathbb{I} \text {. }
$$


Applying Lemma 2.7 to the pair $\left(M_{11}, M_{22}\right)$, Assumption 3.3 implies the existence of matrix functions $S, Z_{1}, Z_{2}$ of appropriate sizes that have the following properties

i) the function $\left[\begin{array}{c}S \\ Z_{1}\end{array}\right] \in C\left(\mathbb{I}, \mathbb{C}^{r, r}\right)$ is pointwise nonsingular,

ii) $Z_{1} M_{11}+Z_{2} M_{22}=0$

iii) the function pair $\left(S M_{11}, M_{22}\right)$ has no hidden redundancy.

Scaling system (3.3) with the function

$$
P_{2}:=\operatorname{diag}\left(\left[\begin{array}{c}
S \\
Z_{1}
\end{array}\right], I_{a}, I_{v}\right) \in C\left(\mathbb{I}, \mathbb{C}^{m, m}\right)
$$

we obtain

$$
\left[\begin{array}{cc}
S M_{11} & S M_{12} \\
Z_{1} M_{11} & Z_{1} M_{12} \\
0 & M_{22} \\
0 & 0
\end{array}\right]\left[\begin{array}{c}
\dot{x} \\
x
\end{array}\right]=\left[\begin{array}{c}
S f_{1} \\
Z_{1} f_{1} \\
f_{2} \\
0
\end{array}\right] \begin{aligned}
& d \\
& a \\
& v
\end{aligned}
$$

where $r=d+s$.

Clearly, one can see that the step of transforming system (3.1) via 3.3 to 3.5 is nothing else than scaling a system with pointwise nonsingular functions, and hence, this preserves the solution set of system (3.1). We then reduce the number of differential equations in (3.5) from $r$ to $d$ by removing the block $Z_{1} M_{11}$ as in the following lemma.

Lemma 3.4. Consider the DAE (3.5), and the matrix-valued functions $S, Z_{1}, Z_{2}$ defined by Lemma 2.7 applies to (3.3). Then, system (3.5) has the same solution set as the equation

$$
\left[\begin{array}{cc}
S M_{11} & S M_{12} \\
0 & Z_{1} M_{12}+Z_{2} \dot{M}_{22} \\
0 & M_{22} \\
0 & 0
\end{array}\right]\left[\begin{array}{l}
\dot{x} \\
x
\end{array}\right]=\left[\begin{array}{c}
S f_{1} \\
Z_{1} f_{1}+Z_{2} \dot{f}_{2} \\
f_{2} \\
0
\end{array}\right] \begin{gathered}
d \\
s \\
a \\
v
\end{gathered}
$$

Consequently, the DAE 1.2 has the same solution set as system (3.6).

Proof. By differentiating the third block equation of (3.5) and scaling it with $Z_{2}$, we obtain

$$
Z_{2} M_{22} \dot{x}+Z_{2} \dot{M}_{22} x=Z_{2} \dot{f}_{2}
$$

We see that the difference between system (3.5) and system (3.6) is only the second block equation, where a differential equation is replaced by an algebraic equation. This step is done by simply adding equation (3.7) to the second block equation of (3.5), and making use of the identity (3.4). Vice versa, one can subtract equation (3.7) from the second block equation of (3.6), and therefore, obtain (3.5). This guarantees that systems 3.5 and 3.6 have the same solution set, and therefore, systems (1.2) and (3.6) also have the same solution set.

In (3.6) the number of differential equations has been reduced to $d=r-s$ and we can continue this reduction process leading to the following procedure.

Procedure 3.5.

Input: $A$ DAE of the form (1.2) and its algebraic form (3.1).

Begin: Set $i=0$ and let $E^{0}=E, A^{0}=A, f^{0}=f, M^{0}=\left[\begin{array}{ll}E^{0} & -A^{0}\end{array}\right]$. 
Step 1. Assume that there exist two integers $r^{i}$, a $a^{i}$ such that

$$
\operatorname{rank}\left(E^{i}\right)=r^{i}, \operatorname{rank}\left(M^{i}\right)=r^{i}+a^{i} \text { for all } t \in \mathbb{I} \text {. }
$$

Determine a pointwise nonsingular function $P_{1} \in C\left(\mathbb{I}, \mathbb{C}^{m, m}\right)$ such that by scaling the equation $M^{i}\left[\begin{array}{l}\dot{x} \\ x\end{array}\right]=f^{i}$ from the left with $P_{1}$ we obtain

$$
\left[\begin{array}{cc}
M_{11} & M_{12} \\
0 & M_{22} \\
0 & 0
\end{array}\right]\left[\begin{array}{l}
\dot{x} \\
x
\end{array}\right]=\left[\begin{array}{l}
f_{1} \\
f_{2} \\
f_{3}
\end{array}\right] \begin{aligned}
& r^{i} \\
& a^{i} \\
& v^{i}
\end{aligned}
$$

with $v^{i}=m-r^{i}-a^{i}$ and where the functions $M_{11} \in C\left(\mathbb{I}, \mathbb{C}^{r^{i}, n}\right)$, and $M_{22} \in C\left(\mathbb{I}, \mathbb{C}^{a^{i}, n}\right)$ have pointwise full row rank.

Step 2. Suppose that there exists an integer $\hat{m}$ such that

$$
\operatorname{rank}\left(\left[\begin{array}{l}
M_{11} \\
M_{22}
\end{array}\right]\right)=\hat{m}, \text { for all } t \in \mathbb{I} .
$$

If the function pair $\left(M_{11}, M_{22}\right)$ has no hidden redundancy, i.e., if $\hat{m}=r^{i}+a^{i}$ then $\boldsymbol{S T O P}$ with the resulting system 3.8 ,

else if $s^{i}=r^{i}+a^{i}-\hat{m}>0$ then proceed to Step 3.

Step 3. Determine functions $S, Z_{1}, Z_{2}$ of appropriate sizes such that

i) the matrix-valued function $\left[\begin{array}{c}S \\ Z_{1}\end{array}\right] \in C\left(\mathbb{I}, \mathbb{C}^{r^{i}, r^{i}}\right)$ is pointwise nonsingular,

ii) $Z_{1} M_{11}+Z_{2} M_{22}=0$,

iii) the function pair $\left(S M_{11}, M_{22}\right)$ has no hidden redundancy.

Set

$$
P_{2}:=\operatorname{diag}\left(\left[\begin{array}{c}
S \\
Z_{1}
\end{array}\right], I_{a^{i}}, I_{v^{i}}\right) \in C\left(\mathbb{I}, \mathbb{C}^{m, m}\right),
$$

and scale system 3.8 with $P_{2}$ from the left to obtain

$$
\left[\begin{array}{cc}
S M_{11} & S M_{12} \\
Z_{1} M_{11} & Z_{1} M_{12} \\
0 & M_{22} \\
0 & 0
\end{array}\right]\left[\begin{array}{l}
\dot{x} \\
x
\end{array}\right]=\left[\begin{array}{c}
S f_{1} \\
Z_{1} f_{1} \\
f_{2} \\
0
\end{array}\right] \begin{aligned}
& d^{i} \\
& s^{i} \\
& v^{i}
\end{aligned}
$$

Step 4. Remove the block $Z_{1} M_{11}$ in system (3.9) (as in Lemma 3.4 to obtain

$$
\left[\begin{array}{cc}
S M_{11} & S M_{12} \\
0 & Z_{1} M_{12}+Z_{2} \dot{M}_{22} \\
0 & M_{22} \\
0 & 0
\end{array}\right]\left[\begin{array}{l}
\dot{x} \\
x
\end{array}\right]=\left[\begin{array}{c}
S f_{1} \\
Z_{1} f_{1}+Z_{2} \dot{f}_{2} \\
f_{2} \\
0
\end{array}\right] \begin{gathered}
d^{i} \\
s^{i} \\
a^{i} \\
v^{i}
\end{gathered}
$$

Increase $i$ by 1 , set

$$
E^{i}:=\left[\begin{array}{c}
S M_{11} \\
0 \\
0 \\
0
\end{array}\right], A^{i}:=-\left[\begin{array}{c}
S M_{12} \\
Z_{1} M_{12}+Z_{2} \dot{M}_{22} \\
M_{22} \\
0
\end{array}\right], f^{i}=\left[\begin{array}{c}
S f_{1} \\
Z_{1} f_{1}+Z_{2} \dot{f}_{2} \\
f_{2} \\
0
\end{array}\right], M^{i}:=\left[\begin{array}{ll}
E^{i} & -A^{i}
\end{array}\right],
$$


and repeat the process from Step 1.

End.

Since $r^{i+1}=d^{i}=r^{i}-s^{i}$, and $s^{i}>0$ for all $i$ except for the last iteration, Procedure 3.5 terminates after a finite number of iterations.

In the following we set $\mu=\min \left\{i \in \mathbb{N}_{0}, s^{i}=0\right\}$, which is a characteristic quantity of the DAE (1.2).

TheOREm 3.6. Consider the DAE 1.2 and assume that Procedure 3.5 proceeds until termination, i.e., all the constant rank assumptions in Procedure 3.5 are fulfilled. Then, system (1.2) has the same solution set as the resulting DAE (3.8) which we denote by

$$
\left[\begin{array}{cc}
\hat{M}_{11} & \hat{M}_{12} \\
0 & \hat{M}_{22} \\
0 & 0
\end{array}\right]\left[\begin{array}{l}
\dot{x} \\
x
\end{array}\right]=\left[\begin{array}{l}
\hat{f}_{1} \\
\hat{f}_{2} \\
\hat{f}_{3}
\end{array}\right]
$$

where $\left[\begin{array}{l}\hat{M}_{11} \\ \hat{M}_{22}\end{array}\right]$ has pointwise full row rank, and the $\hat{f}_{i}(i=1,2,3)$ are functions of $f, \dot{f}, \ldots, f^{(\mu)}$.

Proof. After carrying out Procedure 3.5 , we obtain a system of the form 3.10$)$, where $\hat{M}_{11}, \hat{M}_{22}$ have pointwise full row rank and the pair $\left(\hat{M}_{11}, \hat{M}_{22}\right)$ has no hidden redundancy. By definition, this means that

$$
\operatorname{rank}\left(\left[\begin{array}{c}
\hat{M}_{11} \\
\hat{M}_{22}
\end{array}\right]\right)=\operatorname{rank}\left(\hat{M}_{11}\right)+\operatorname{rank}\left(\hat{M}_{22}\right)
$$

and hence, $\left[\begin{array}{l}\hat{M}_{11} \\ \hat{M}_{22}\end{array}\right]$ has pointwise full row rank. Furthermore, due to Lemma 3.4 the solution set of system 1.2 is preserved in every step, thus it follows that 1.2 and 3.10 have the same solution set.

3.2. Difference equations. Analogous to the case of differential-algebraic equations, we may also treat the difference equation $(1.3)$ via an algebraic method. Here we propose, without proof, modifications of Procedure 3.5 and of Theorem 3.6 for the case of difference equations.

Procedure 3.7.

Input: A difference equation of the form 1.3 expressed in the algebraic form

$$
\left[\begin{array}{ll}
A & B
\end{array}\right]\left[\begin{array}{c}
x \\
\Delta_{\tau} x
\end{array}\right]=-f
$$

in the time interval $\mathbb{I}$.

Begin: Set $i=0$ and let $A^{0}:=A, B^{0}:=B, f^{0}:=f, W^{0}:=\left[\begin{array}{ll}A^{0} & B^{0}\end{array}\right]$.

Step 1. Assume that there exist two integers $w_{1}^{i}$, w $w_{2}^{i}$ such that

$$
\operatorname{rank}\left(A^{i}\right)=w_{1}^{i}, \operatorname{rank}\left(W^{i}\right)=w_{1}^{i}+w_{2}^{i}, \text { for all } t \in \mathbb{I} .
$$

Determine a pointwise nonsingular function $P_{1} \in C\left(\mathbb{I}, \mathbb{C}^{m, m}\right)$ and scale the equation $W^{i}\left[\begin{array}{c}x \\ \Delta_{\tau} x\end{array}\right]=$ 
$-f^{i}$ with $P_{1}$ to obtain

$$
\left[\begin{array}{cc}
W_{11} & W_{12} \\
0 & W_{22} \\
0 & 0
\end{array}\right]\left[\begin{array}{c}
x \\
\Delta_{\tau} x
\end{array}\right]=-\left[\begin{array}{l}
f_{1} \\
f_{2} \\
f_{3}
\end{array}\right] \begin{gathered}
w_{1}^{i} \\
w_{2}^{i}, \\
w_{3}^{i}
\end{gathered}
$$

with $w_{3}^{i}=m-w_{1}^{i}-w_{2}^{i}$ and where the functions $W_{11} \in C\left(\mathbb{I}, \mathbb{C}^{w_{1}^{i}, n}\right)$, and $W_{22} \in C\left(\mathbb{I}, \mathbb{C}^{w_{2}^{i}, n}\right)$ have pointwise full row rank.

Step 2. Suppose that there exists an integer $\widetilde{w}$ such that

$$
\operatorname{rank}\left(\left[\begin{array}{c}
W_{11} \\
\Delta_{-\tau} W_{22}
\end{array}\right]\right)=\widetilde{w}, \text { for all } t \in \mathbb{I} \text {. }
$$

If the pair $\left(W_{11}, \Delta_{-\tau} W_{22}\right)$ has no hidden redundancy, i.e., if $\widetilde{w}=w_{1}^{i}+w_{2}^{i}$, then $\boldsymbol{S T O P}$ with the resulting system (3.11),

else if $s^{i}=w_{1}^{i}+w_{2}^{i}-\widetilde{w}>0$ then proceed to Step 3 .

Step 3. Determine functions $S, Z_{1}, Z_{2}$ of appropriate sizes such that
i) the matrix-valued function $\left[\begin{array}{c}S \\ Z_{1}\end{array}\right] \in C\left(\mathbb{I}, \mathbb{C}^{w_{1}^{i}, w_{1}^{i}}\right)$ is pointwise nonsingular,
ii) $Z_{1} W_{11}+Z_{2} \Delta_{-\tau} W_{22}=0$
iii) the function pair $\left(S W_{11}, \Delta_{-\tau} W_{22}\right)$ has no hidden redundancy.

Set $P_{2}:=\operatorname{diag}\left(\left[\begin{array}{c}S \\ Z_{1}\end{array}\right], I_{w_{2}^{i}}, I_{w_{3}^{i}}\right) \in C\left(\mathbb{I}, \mathbb{C}^{m, m}\right)$, and scale system 3.11 from the left by $P_{2}$ to obtain

$$
\left[\begin{array}{cc}
S W_{11} & S W_{12} \\
Z_{1} W_{11} & Z_{1} W_{12} \\
0 & W_{22} \\
0 & 0
\end{array}\right]\left[\begin{array}{c}
x \\
\Delta_{\tau} x
\end{array}\right]=-\left[\begin{array}{c}
S f_{1} \\
Z_{1} f_{1} \\
f_{2} \\
0
\end{array}\right] \begin{gathered}
d^{i} \\
s^{i} \\
w_{2}^{i} \\
w_{3}^{i}
\end{gathered}
$$

where $w_{1}^{i}=d^{i}+s^{i}$.

Step 4. Shift forward the third block equation of system (3.13), and then scale it with $Z_{2}$ from the left to obtain

$$
Z_{2} \Delta_{-\tau} W_{22} x=-Z_{2} \Delta_{-\tau} f_{2} .
$$

Add this equation to the second block equation of (3.13), and use the identity (3.12) to obtain

$$
\left[\begin{array}{cc}
S W_{11} & S W_{12} \\
0 & Z_{1} W_{12} \\
0 & W_{22} \\
0 & 0
\end{array}\right]\left[\begin{array}{c}
x \\
\Delta_{\tau} x
\end{array}\right]=-\left[\begin{array}{c}
S f_{1} \\
Z_{1} f_{1}+Z_{2} \Delta_{-\tau} f_{2} \\
f_{2} \\
0
\end{array}\right]
$$

Increase $i$ by 1 , set

$$
A^{i}:=\left[\begin{array}{c}
S W_{11} \\
0 \\
0 \\
0
\end{array}\right], B^{i}:=\left[\begin{array}{c}
S W_{12} \\
Z_{1} W_{12} \\
W_{22} \\
0
\end{array}\right], f^{i}:=\left[\begin{array}{c}
S f_{1} \\
Z_{1} f_{1}+Z_{2} \Delta_{-\tau} f_{2} \\
f_{2} \\
0
\end{array}\right], W^{i}:=\left[\begin{array}{ll}
A^{i} & B^{i}
\end{array}\right],
$$


and repeat the process from Step 1.

\section{End.}

Analogous to Procedure 3.5. Procedure 3.7 applied to the difference equation 1.3 will terminate after a finite number of steps, which immediately leads to the following theorem.

TheOREM 3.8. Consider the difference equation (1.3) and assume that Procedure 3.7 proceeds until termination, i.e., all the constant rank assumptions in Procedure 3.7 are fulfilled. Then, (1.3) has the same solution set as the resulting difference equation 3.11] which we will denote by

$$
\left[\begin{array}{cc}
\widehat{W}_{11} & \widehat{W}_{12} \\
0 & \widehat{W}_{22} \\
0 & 0
\end{array}\right]\left[\begin{array}{c}
x \\
\Delta_{\tau} x
\end{array}\right]=-\left[\begin{array}{l}
\hat{f}_{1} \\
\hat{f}_{2} \\
\hat{f}_{3}
\end{array}\right] \begin{aligned}
& \hat{w}_{1} \\
& \hat{w}_{2} \\
& \hat{w}_{3}
\end{aligned}
$$

where the function $\left[\begin{array}{c}\widehat{W}_{11} \\ \Delta_{-\tau} \widehat{W}_{22}\end{array}\right]: t \mapsto\left[\begin{array}{c}\widehat{W}_{11} \\ \widehat{W}_{22}(t+\tau)\end{array}\right]$ has pointwise full row rank. The size of the block row equations are $\hat{w}_{1}, \hat{w}_{2}$ and $\hat{w}_{3}=m-\hat{w}_{1}-\hat{w}_{2}$.

It will turn out later that during our reformulation procedure for general delay differentialalgebraic equations, sometimes we need to differentiate difference equations, but it is possible that some components of the function $\Delta_{\tau} x$ may not be differentiable, and therefore differentiating an equation that does not contain $\Delta_{\tau} x$ is more advantageous than differentiating one that contains it. Thus, we suggest to use a scaling, e.g. via Gaussian elimination, to reduce the number of difference equations that involve $\Delta_{\tau} x$ in system (3.14) as in the following corollary.

Corollary 3.9. Consider the difference equation (1.3) and the resulting system (3.14) obtained by applying Procedure 3.7 to (1.3). Furthermore, suppose that in system (3.14) the pair $\left(\widehat{W}_{12}, \widehat{W}_{22}\right)$ satisfies

$$
\operatorname{rank}\left(\left[\begin{array}{c}
\widehat{W}_{12} \\
\widehat{W}_{22}
\end{array}\right]\right)=\hat{w}_{4}, \text { for all } t \in \mathbb{I},
$$

for some integer $\hat{w}_{4}$. Then, there exist a pointwise nonsingular matrix-valued function $P_{3} \in$ $C\left(\mathbb{I}, \mathbb{C}^{m, m}\right)$ such that by scaling system (3.14) with $P_{3}$ from the left one obtains

$$
\left[\begin{array}{cc}
\widetilde{W}_{11} & \widetilde{W}_{12} \\
0 & \widehat{W}_{22} \\
0 & 0
\end{array}\right]\left[\begin{array}{c}
x \\
\Delta_{\tau} x
\end{array}\right]=-\left[\begin{array}{l}
\tilde{f}_{1} \\
\hat{f}_{2} \\
\hat{f}_{3}
\end{array}\right] \begin{aligned}
& \hat{w}_{1} \\
& \hat{w}_{2} \\
& \hat{w}_{3}
\end{aligned}
$$

where the function $\left[\begin{array}{c}\widetilde{W}_{11} \\ \Delta_{-\tau} \widehat{W}_{22}\end{array}\right]$ has pointwise full row rank, and the pair $\left(\widetilde{W}_{12}, \widehat{W}_{22}\right)$ has no hidden redundancy.

Proof. Applying Lemma 2.7 to the pair $\left(\widehat{W}_{12}, \widehat{W}_{22}\right)$, we can determine matrix-valued functions $\widehat{S}, \widehat{Z}_{1}, \widehat{Z}_{2}$ of appropriate sizes that have the following properties

i) the function $\left[\begin{array}{c}\widehat{S} \\ \widehat{Z}_{1}\end{array}\right] \in C\left(\mathbb{I}, \mathbb{C}^{\hat{w}_{1}, \hat{w}_{1}}\right)$ is pointwise nonsingular,

ii) $\widehat{Z}_{1} \widehat{W}_{12}+\widehat{Z}_{2} \widehat{W}_{22}=0$,

iii) the function $\widehat{S} W_{12}$ has pointwise full row rank, and the pair $\left(\widehat{S}_{12}, \widehat{W}_{22}\right)$ has no hidden redundancy. 
Set

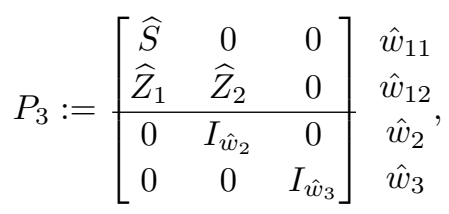

where $\hat{w}_{1}=\hat{w}_{11}+\hat{w}_{12}$, and scale system (3.14) from the left by $P_{3}$ to obtain

$$
\left[\begin{array}{c|c}
\widehat{S} \widehat{W}_{11} & \widehat{S W}_{12} \\
\widehat{Z}_{1} \widehat{W}_{11} & 0 \\
\hline 0 & \widehat{W}_{22} \\
0 & 0
\end{array}\right]\left[\begin{array}{c}
x \\
\Delta_{\tau} x
\end{array}\right]=-\left[\begin{array}{c}
\widehat{S} \hat{f}_{1} \\
\widehat{Z}_{1} \hat{f}_{1}+\widehat{Z}_{2} \hat{f}_{2} \\
\hat{f}_{2} \\
\hat{f}_{3}
\end{array}\right] \begin{gathered}
\hat{w}_{11} \\
\hat{w}_{12} \\
\hat{w}_{2} \\
\hat{w}_{3}
\end{gathered}
$$

Setting

$$
\widetilde{W}_{11}:=\left[\begin{array}{c}
\widehat{S} \\
\widehat{Z}_{1}
\end{array}\right] \widehat{W}_{11}, \widetilde{W}_{12}:=\left[\begin{array}{c}
\widehat{S} \widehat{W}_{11} \\
0
\end{array}\right], \tilde{f}_{1}:=\left[\begin{array}{c}
\widehat{S} \hat{f}_{1} \\
\widehat{Z}_{1} \hat{f}_{1}+\widehat{Z}_{2} \hat{f}_{2}
\end{array}\right],
$$

we obtain the desired form 3.15 .

The remaining part now is to prove that in system $\left[3.15\right.$, the function $\left[\begin{array}{c}\widetilde{W}_{11} \\ \Delta_{-\tau} \widehat{W}_{22}\end{array}\right]$ has pointwise full row rank. Note that the pair $\left(\widetilde{W}_{11}, \Delta_{-\tau} \widehat{W}_{22}\right)$ has no hidden redundancy due to Lemma 2.6 . and the two functions $\widetilde{W}_{11}, \widehat{W}_{22}$ have pointwise full row rank. Therefore, also $\left[\begin{array}{c}\widetilde{W}_{11} \\ \Delta_{-\tau} \widehat{W}_{22}\end{array}\right]$ has pointwise full row rank.

By transforming system (3.14 to (3.16), we have reduced the number of difference equations that contain $\Delta_{\tau} x$ from $\hat{w}_{1}+\hat{w}_{2}$ to $\hat{w}_{11}+\hat{w}_{2}$. Furthermore, since the function $\widehat{S}_{12}$ has pointwise full row rank, we see that it is not possible to reduce any further by scaling, e.g. via Gaussian elimination.

4. Analysis of general linear DDAEs. This section discusses DDAEs with a single delay of the form (1.1) and the initial value problem (1.1), (1.5). First, we study a reformulation procedure to transform the DDAE 1.1 into another system, where all hidden constraints and solvability conditions can be read off.

As we have seen in Section 3 , in order to study differential-algebraic (resp., difference) equations, it is possible to use differentiation (resp., shift) operators as well as scaling to transform a given system. Thus, for an arbitrary linear DDAE, in general we can use the following three operations

i) scaling the system with a pointwise nonsingular matrix-valued function,

ii) adding to one equation derivatives of other equations,

iii) shifting forward equations that contain only $\Delta_{\tau} x(t)$ but not $x(t)$.

Previous work about DDAEs typically considered only the first two operations, see e.g. 2, 6, 7, 8, which are based on the assumption that the associated DAE

$$
E(t) \dot{x}(t)=A(t) x(t)+g(t),
$$

has a unique solution for every smooth enough inhomogeneity $g$. In this case, the DDAE (1.1) can be interpreted as a DAE 4.1a by introducing a new inhomogeneity

$$
g(t):=B(t) \Delta_{\tau} x(t)+f(t) .
$$


In this special case with a prescribed past function $\Delta_{\tau} x(t)$ on the interval $[(k-1) \tau, k \tau], k \in \mathbb{N}_{0}$, one can solve $\left.x\right|_{[k \tau,(k+1) \tau]}$ from the DAE (4.1a), and consequently the function $x$ will be determined recursively by solving a sequence of DAEs.

However, this method is no longer appropriate for general DDAEs, where the associated DAE 4.1a is over- or underdetermined. This fact can be easily seen from the trivial equation

$$
0 \cdot \dot{x}(t)=0 \cdot x(t)+x(t-1)-t, t \in \mathbb{I} .
$$

Moreover, we also demonstrate in the following example that the two operations differentiation and shifting do not commute, so the order of their use is crucial. In fact, choosing a wrong order of those operations may lead to additional (but unnecessary) smoothness requirements.

EXAmple 4.1. Consider the DDAE

$$
\left[\begin{array}{lll}
1 & 0 & 0 \\
0 & 0 & 0 \\
0 & 0 & 0
\end{array}\right] \dot{x}(t)=\left[\begin{array}{ccc}
0 & 0 & 0 \\
1 & 0 & 0 \\
0 & 0 & 0
\end{array}\right] x(t)+\left[\begin{array}{ccc}
0 & 0 & 1 \\
0 & 1 & 0 \\
1 & 0 & 0
\end{array}\right] \Delta_{\tau} x(t)+\left[\begin{array}{l}
f_{1}(t) \\
f_{2}(t) \\
f_{3}(t)
\end{array}\right]
$$

in the interval $t \in \mathbb{I}$.

If one starts with a shift operation first, then shifting the last equation of 4.2 leads to

$$
\left[\begin{array}{lll}
1 & 0 & 0 \\
0 & 0 & 0 \\
0 & 0 & 0
\end{array}\right] \dot{x}(t)=\left[\begin{array}{lll}
0 & 0 & 0 \\
1 & 0 & 0 \\
1 & 0 & 0
\end{array}\right] x(t)+\left[\begin{array}{lll}
0 & 0 & 1 \\
0 & 1 & 0 \\
0 & 0 & 0
\end{array}\right] \Delta_{\tau} x(t)+\left[\begin{array}{c}
f_{1}(t) \\
f_{2}(t) \\
f_{3}(t+\tau)
\end{array}\right] .
$$

Adding the first derivative of the last equation to the first equation and removing the term $\dot{x}_{1}$ on both sides, one obtains

$$
\left[\begin{array}{l}
0 \\
0 \\
0
\end{array}\right]=\left[\begin{array}{lll}
0 & 0 & 0 \\
1 & 0 & 0 \\
1 & 0 & 0
\end{array}\right] x(t)+\left[\begin{array}{lll}
0 & 0 & 1 \\
0 & 1 & 0 \\
0 & 0 & 0
\end{array}\right] \Delta_{\tau} x(t)+\left[\begin{array}{c}
f_{1}(t)+\dot{f}_{3}(t+\tau) \\
f_{2}(t) \\
f_{3}(t+\tau)
\end{array}\right] .
$$

Subtracting the last equation from the second one leads to

$$
\left[\begin{array}{l}
0 \\
0 \\
0
\end{array}\right]=\left[\begin{array}{lll}
0 & 0 & 0 \\
0 & 0 & 0 \\
1 & 0 & 0
\end{array}\right] x(t)+\left[\begin{array}{lll}
0 & 0 & 1 \\
0 & 1 & 0 \\
0 & 0 & 0
\end{array}\right] \Delta_{\tau} x(t)+\left[\begin{array}{c}
f_{1}(t)+\dot{f}_{3}(t+\tau) \\
f_{2}(t)-f_{3}(t+\tau) \\
f_{3}(t+\tau)
\end{array}\right] .
$$

Shifting the first two equations, we obtain the system

$$
\left[\begin{array}{l}
0 \\
0 \\
0
\end{array}\right]=\left[\begin{array}{lll}
0 & 0 & 1 \\
0 & 1 & 0 \\
1 & 0 & 0
\end{array}\right] x(t)+\left[\begin{array}{c}
f_{1}(t+\tau)+\dot{f}_{3}(t+2 \tau) \\
f_{2}(t+\tau)-f_{3}(t+2 \tau) \\
f_{3}(t+\tau)
\end{array}\right]
$$

Proceeding this way (using the shift operation first) only 2 shifts and 1 differentiation are used, and more important, only $f_{3}$ is required to be continuously differentiable. We now demonstrate that if one starts with a differentiation first, then unnecessary smoothness requirements for $f$ follow.

First adding the first derivative of the second equation to the first equation of 4.2 and removing the term $\dot{x}_{1}$ on both sides leads to

$$
\left[\begin{array}{l}
0 \\
0 \\
0
\end{array}\right]=\left[\begin{array}{lll}
0 & 0 & 0 \\
1 & 0 & 0 \\
0 & 0 & 0
\end{array}\right] x(t)+\left[\begin{array}{lll}
0 & 0 & 1 \\
0 & 1 & 0 \\
1 & 0 & 0
\end{array}\right] \Delta_{\tau} x(t)+\left[\begin{array}{lll}
0 & 1 & 0 \\
0 & 0 & 0 \\
0 & 0 & 0
\end{array}\right] \Delta_{\tau} \dot{x}(t)+\left[\begin{array}{c}
f_{1}(t)+\dot{f}_{2}(t) \\
f_{2}(t) \\
f_{3}(t)
\end{array}\right] .
$$


Shifting the first and the last equation, one has

$$
\left[\begin{array}{ccc}
0 & -1 & 0 \\
0 & 0 & 0 \\
0 & 0 & 0
\end{array}\right] \dot{x}(t)=\left[\begin{array}{ccc}
0 & 0 & 1 \\
1 & 0 & 0 \\
1 & 0 & 0
\end{array}\right] x(t)+\left[\begin{array}{ccc}
0 & 0 & 0 \\
0 & 1 & 0 \\
0 & 0 & 0
\end{array}\right] \Delta_{\tau} x(t)+\left[\begin{array}{c}
f_{1}(t+\tau)+\dot{f}_{2}(t+\tau) \\
f_{2}(t) \\
f_{3}(t+\tau)
\end{array}\right] .
$$

Subtracting the last equation from the second one leads to

$$
\left[\begin{array}{ccc}
0 & -1 & 0 \\
0 & 0 & 0 \\
0 & 0 & 0
\end{array}\right] \dot{x}(t)=\left[\begin{array}{ccc}
0 & 0 & 1 \\
0 & 0 & 0 \\
1 & 0 & 0
\end{array}\right] x(t)+\left[\begin{array}{ccc}
0 & 0 & 0 \\
0 & 1 & 0 \\
0 & 0 & 0
\end{array}\right] \Delta_{\tau} x(t)+\left[\begin{array}{c}
f_{1}(t+\tau)+\dot{f}_{2}(t+\tau) \\
f_{2}(t)-f_{3}(t+\tau) \\
f_{3}(t+\tau)
\end{array}\right] .
$$

Shifting the second equation, one then gets

$$
\left[\begin{array}{ccc}
0 & -1 & 0 \\
0 & 0 & 0 \\
0 & 0 & 0
\end{array}\right] \dot{x}(t)=\left[\begin{array}{ccc}
0 & 0 & 1 \\
0 & 1 & 0 \\
1 & 0 & 0
\end{array}\right] x(t)+\left[\begin{array}{ccc}
0 & 0 & 0 \\
0 & 0 & 0 \\
0 & 0 & 0
\end{array}\right] \Delta_{\tau} x(t)+\left[\begin{array}{c}
f_{1}(t+\tau)+\dot{f}_{2}(t+\tau) \\
f_{2}(t+\tau)-f_{3}(t+2 \tau) \\
f_{3}(t)
\end{array}\right] \text {. }
$$

Subtracting the first derivative of the second equation from the first one and removing the term $\dot{x}_{2}(t)$ on both sides will also lead to system 4.3 . However, we have used two differentiations and two shifts, and more important, during this process the differentiability of not only $f_{3}(t)$ but also of $f_{2}(t)$ is required, which is unnecessary.

Beside demonstrating the non-commutativity of the differentiation and shift operations, Example 4.1 also suggests that one should avoid as much as possible to differentiate equations that contain the term $\Delta_{\tau} x(t)$. Therefore, before applying a differentiation, one should reduce the number of difference equations that contain $\Delta_{\tau} x(t)$ to minimum. This step can be easily performed using Corollary 3.9 .

Throughout this section, we assume that Procedures 3.5 and 3.7 are applicable so that we are able to remove the hidden redundancy in the difference and differential-algebraic parts of system (1.1). Again, for notational convenience, we omit the time-variable $t$ in all matrix-valued and vector-valued functions.

First we will remove the hidden redundancy in the difference part of system (1.1), using the following procedure.

Procedure 4.2.

Input: A DDAE of the form 1.1

Step 1. Assume that the function $E$ has constant rank, i.e.,

$$
\operatorname{rank}(E)=r, \text { for all } t \in \mathbb{I} .
$$

Determine a pointwise non-singular function $P \in C\left(\mathbb{I}, \mathbb{C}^{m, m}\right)$ and scale system (1.1) with $P$ from the left to obtain

$$
\left[\begin{array}{c}
E_{1} \\
0
\end{array}\right] \dot{x}=\left[\begin{array}{c}
A_{1} \\
A_{2}
\end{array}\right] x+\left[\begin{array}{c}
B_{1} \\
B_{2}
\end{array}\right] \Delta_{\tau} x+\left[\begin{array}{c}
f_{1} \\
f_{2}
\end{array}\right],
$$

where $E_{1}$ has pointwise full row rank $r$.

Step 2. Apply Procedure 3.7 to the lower block equation of system 4.4 to obtain

$$
\left[\begin{array}{c}
E_{1} \\
0 \\
0 \\
0
\end{array}\right] \dot{x}=\left[\begin{array}{c}
A_{1} \\
\breve{A}_{2} \\
0 \\
0
\end{array}\right] x+\left[\begin{array}{c}
B_{1} \\
\breve{B}_{2} \\
\breve{B}_{3} \\
0
\end{array}\right] \Delta_{\tau} x+\left[\begin{array}{c}
f_{1} \\
\breve{f}_{2} \\
\breve{f}_{3} \\
\breve{f}_{4}
\end{array}\right]
$$


where the functions $E_{1},\left[\begin{array}{c}\breve{A}_{2} \\ \Delta_{-\tau} \breve{B}_{3}\end{array}\right]$ have pointwise full row rank.

Step 3. Apply Corollary 3.9 to the difference equation consisting of the second and the third block row equations of system 4.5 to obtain

$$
\left[\begin{array}{c}
E_{1} \\
0 \\
0 \\
0
\end{array}\right] \dot{x}=\left[\begin{array}{c}
A_{1} \\
\widetilde{A}_{2} \\
0 \\
0
\end{array}\right] x+\left[\begin{array}{c}
B_{1} \\
\widetilde{B}_{2} \\
\widetilde{B}_{3} \\
0
\end{array}\right] \Delta_{\tau} x+\left[\begin{array}{c}
f_{1} \\
\tilde{f}_{2} \\
\tilde{f}_{3} \\
\tilde{f}_{4}
\end{array}\right]
$$

where the functions $E_{1},\left[\begin{array}{c}\widetilde{A}_{2} \\ \Delta_{-\tau} \widetilde{B}_{3}\end{array}\right]$ have pointwise full row rank, and $\left(\widetilde{B}_{2}, \widetilde{B}_{3}\right)$ has no hidden redundancy.

\section{End.}

We now turn to the step of removing the hidden redundancy in the differential-algebraic part. In order to do that, we perform the following procedure.

PROCEDURE 4.3.

Input: A DDAE of the form 4.6 where the functions $E_{1},\left[\begin{array}{c}\widetilde{A}_{2} \\ \Delta_{-\tau} \widetilde{B}_{3}\end{array}\right]$ have pointwise full row rank, and $\left(\widetilde{B}_{2}, \widetilde{B}_{3}\right)$ has no hidden redundancy.

Step 1. Shift forward the third block equation of system [4.6] to obtain

$$
\underbrace{\left[\begin{array}{c}
E_{1} \\
0 \\
0 \\
0
\end{array}\right]}_{\widetilde{E}} \dot{x}=\underbrace{\left[\begin{array}{c}
A_{1} \\
\widetilde{A}_{2} \\
\Delta_{-\tau} \widetilde{B}_{3} \\
0
\end{array}\right]}_{\widetilde{A}} x+\underbrace{\left[\begin{array}{c}
B_{1} \\
\widetilde{B}_{2} \\
0 \\
0
\end{array}\right]}_{\widetilde{B}} \Delta_{\tau} x+\underbrace{\left[\begin{array}{c}
f_{1} \\
\tilde{f}_{2} \\
\Delta_{-\tau} \tilde{f}_{3} \\
\tilde{f}_{4}
\end{array}\right]}_{\tilde{f}},
$$

where the functions $E_{1},\left[\begin{array}{c}\widetilde{A}_{2} \\ \Delta_{-\tau} \widetilde{B}_{3}\end{array}\right]$ have pointwise full row rank.

Step 2. Let $\tilde{g}:=\widetilde{B} \Delta_{\tau} x+\tilde{f}$, and rewrite system 4.7 in the differential-algebraic form

$$
\widetilde{E} \dot{x}=\widetilde{A} x+\tilde{g} \Leftrightarrow\left[\begin{array}{c}
E_{1} \\
0 \\
0 \\
0
\end{array}\right] \dot{x}=\left[\begin{array}{c}
A_{1} \\
\widetilde{A}_{2} \\
\Delta_{-\tau} \widetilde{B}_{3} \\
0
\end{array}\right] x+\left[\begin{array}{c}
\tilde{g}_{1} \\
\tilde{g}_{2} \\
\Delta_{-\tau} \tilde{f}_{3} \\
\tilde{f}_{4}
\end{array}\right] .
$$

Step 3. Apply Procedure 3.5 to remove the hidden redundancy of the pair $(\widetilde{E}, \widetilde{A})$ in system 4.8).

Step 4. Shift backward the block equation

$$
0=\Delta_{-\tau} \widetilde{B}_{3} x+\Delta_{-\tau} \tilde{f}_{3} .
$$

in the system achieved from Step 3 to obtain the resulting system

$$
\left[\begin{array}{c}
\hat{E}_{1} \\
0 \\
0 \\
0 \\
0
\end{array}\right] \dot{x}=\left[\begin{array}{c}
\hat{A}_{1} \\
\hat{A}_{2} \\
0 \\
0 \\
0
\end{array}\right] x+\left[\begin{array}{c}
\hat{B}_{1,0} \\
\hat{B}_{2,0} \\
\widetilde{B}_{3} \\
\hat{B}_{3,0} \\
0
\end{array}\right] \Delta_{\tau} x+\sum_{i=1}^{\mu}\left[\begin{array}{c}
0 \\
\hat{B}_{2, i} \\
0 \\
\hat{B}_{3, i} \\
0
\end{array}\right] \Delta_{\tau} x^{(i)}+\left[\begin{array}{c}
\hat{f}_{1} \\
\hat{f}_{2} \\
\tilde{f}_{3} \\
\hat{f}_{3} \\
\tilde{f}_{4}
\end{array}\right] \text {, }
$$


where the matrix-valued function $\left[\begin{array}{llll}\hat{E}_{1}^{T} & \hat{A}_{2}^{T} & \Delta_{-\tau} \widetilde{B}_{3}^{T}\end{array}\right]^{T}$ has pointwise full row rank.

\section{End.}

Due to the observation made in Example 4.1, we first apply Procedure 4.2 and then Procedure 4.3 to system (1.1) to obtain the following lemma.

Lemma 4.4. Consider the application of Procedures 4.2 and then 4.3 to the DDAE (1.1). Then, system 1.1] has the same solution set as system 4.10].

Proof. First applying Procedure 4.2 to system (1.1), we obtain a resulting system (4.6), where the functions $E_{1},\left[\begin{array}{c}\widetilde{A}_{2} \\ \Delta_{-\tau} \widetilde{B}_{3}\end{array}\right]$ have pointwise full row rank, and the pair $\left(\widetilde{B}_{2}, \widetilde{B}_{3}\right)$ has no hidden redundancy. By introducing a new inhomogeneity $\tilde{g}$ as in Step 2 of Procedure 4.3. system (4.7) can be rewritten in differential-algebraic form (4.8).

Step 3 of Procedure 4.3 followed by applying Procedure 3.5 to 4.8 , this step will preserve the three lower block row equations, and only transform the first block row equation into

$$
\left[\begin{array}{c}
\hat{E}_{1} \\
0 \\
0
\end{array}\right] \dot{x}=\left[\begin{array}{c}
\hat{A}_{1} \\
\breve{A}_{2} \\
0
\end{array}\right] x+\left[\begin{array}{l}
\tilde{g}_{11} \\
\tilde{g}_{12} \\
\tilde{g}_{13}
\end{array}\right]
$$

where $\left[\begin{array}{ccccc}\hat{E}_{1}^{T} & \breve{A}_{2}^{T} & \widetilde{A}_{2}^{T} & \Delta_{-\tau} \widetilde{B}_{3}^{T}\end{array}\right]^{T}$ has pointwise full row rank. Let $\mu$ be the characteristic number of the DAE 4.8, then Theorem 3.6 implies that $\tilde{g}_{11}, \tilde{g}_{12}, \tilde{g}_{13}$ are functions of $\tilde{g}, \dot{\tilde{g}}, \ldots, \tilde{g}^{(\mu)}$. More precisely, by resubstituting $\tilde{g}$ by $\tilde{B} \Delta_{\tau} x+\tilde{f}, 4.11$ becomes

$$
\left[\begin{array}{c}
\hat{E}_{1} \\
0 \\
0
\end{array}\right] \dot{x}=\left[\begin{array}{c}
\hat{A}_{1} \\
\hat{H}_{2} \\
0
\end{array}\right] x+\left[\begin{array}{c}
\hat{B}_{1,0} \\
\hat{G}_{2,0} \\
\hat{B}_{3,0}
\end{array}\right] \Delta_{\tau} x+\sum_{i=1}^{\mu}\left[\begin{array}{c}
0 \\
\hat{G}_{2, i} \\
\hat{B}_{3, i}
\end{array}\right] \Delta_{\tau} x^{(i)}+\left[\begin{array}{c}
\hat{f}_{1} \\
\hat{h}_{2} \\
\hat{f}_{3}
\end{array}\right] .
$$

Combining system 4.12 with three lower block row equations of (4.8), setting

$$
\hat{A}_{2}:=\left[\begin{array}{c}
\hat{H}_{2} \\
\widetilde{A}_{2}
\end{array}\right], \hat{f}_{2}:=\left[\begin{array}{c}
\hat{h}_{2} \\
\tilde{f}_{2}
\end{array}\right], \hat{B}_{2,0}:=\left[\begin{array}{c}
\hat{G}_{2,0} \\
\widetilde{B}_{2}
\end{array}\right], \hat{B}_{2, i}:=\left[\begin{array}{c}
\hat{G}_{2, i} \\
0
\end{array}\right], \text { for all } i \geq 1,
$$

and shifting backward the third block row equation of system (4.8), we then get the formulation 4.10.

Remark 4.5. Note that in the last step of Procedure 4.3 we must shift backward the block equation 4.9 because of the following reasons:

i) First, one may need to continue by applying Procedure 4.2 , and therefore, must remove as much as possible all the difference equations that contain $\Delta_{\tau} x$.

ii) The block equation 4.9 does not carry any information about the consistency requirement of an initial function $\phi$, which is properly obtained from the block equation

$$
0=\widetilde{B}_{3} \Delta_{\tau} x+\tilde{f}_{3} .
$$

Introducing

$$
\begin{aligned}
& \mathbb{M}_{0}:=\left\{x: \mathbb{I}_{\tau} \rightarrow \mathbb{C}^{n} \mid \widetilde{B}_{3} \Delta_{\tau} x+\tilde{f}_{3}=0, t \in \mathbb{I}\right\}, \\
& \widetilde{\mathbb{M}}_{0}:=\left\{x \in \mathbb{M}_{0} \mid \sum_{i=0}^{\mu} \hat{B}_{3, i} \Delta_{\tau} x^{(i)}+\hat{f}_{3}=0, t \in \mathbb{I}\right\},
\end{aligned}
$$


we see that $\mathbb{M}_{0}$ (resp., $\widetilde{\mathbb{M}}_{0}$ ) are described by the set of constraints for $\Delta_{\tau} x$ before (resp., after) applying Procedure 4.3 , and $\widetilde{\mathbb{M}}_{0}$ is a subset of $\mathbb{M}_{0}$.

Let us consider the special case of system 4.10 , where $\mathbb{M}_{0}=\widetilde{\mathbb{M}}_{0}$, i.e., the fourth block row equation of system 4.10 is redundant to the third block row equation.

THeOREM 4.6. Consider the DDAE (1.1), its reformulation $(4.10)$, and the sets $\mathbb{M}_{0}, \widetilde{\mathbb{M}}_{0}$ defined by 4.13. Moreover, assume that $\mathbb{M}_{0}=\widetilde{\mathbb{M}}_{0}$. Then 4.10) (and therefore, 1.1) has the same solution set as the system

$$
\left[\begin{array}{c}
\hat{E}_{1} \\
0 \\
0 \\
0
\end{array}\right] \dot{x}=\left[\begin{array}{c}
\hat{A}_{1} \\
\hat{A}_{2} \\
0 \\
0
\end{array}\right] x+\left[\begin{array}{c}
\hat{B}_{1,0} \\
\hat{B}_{2,0} \\
\widetilde{B}_{3} \\
0
\end{array}\right] \Delta_{\tau} x+\sum_{i=1}^{\mu}\left[\begin{array}{c}
0 \\
\hat{B}_{2, i} \\
0 \\
0
\end{array}\right] \Delta_{\tau} x^{(i)}+\left[\begin{array}{c}
\hat{f}_{1} \\
\hat{f}_{2} \\
\tilde{f}_{3} \\
\tilde{f}_{4}
\end{array}\right] .
$$

Here, in 4.14 the function $\left[\begin{array}{llll}\hat{E}_{1}^{T} & \hat{A}_{2}^{T} & \Delta_{-\tau} \widetilde{B}_{3}^{T}\end{array}\right]^{T}$ has pointwise full row rank.

Proof. Since $\mathbb{M}_{0}=\widetilde{\mathbb{M}}_{0}$, every function $x: \mathbb{I}_{\tau} \rightarrow \mathbb{C}^{n}$ that satisfies the third equation of 4.10 will automatically fulfill the fourth equation. Thus, we can remove the fourth equation of system 4.10 to obtain system 4.14.

Let us mention two important consequences of Theorem 4.6, which characterize the structural properties of system (1.1).

Corollary 4.7. Consider the DDAE 1.1] and its reformulation 4.10). Moreover, suppose that the sets $\mathbb{M}_{0}$ and $\widetilde{\mathbb{M}}_{0}$ defined by 4.13 coincide. Then, the following assertions hold.

i) System (1.1) is solvable if and only if in system (4.10) $\tilde{f}_{4}=0$.

ii) System (1.1) is regular if and only if in addition the function $\left[\begin{array}{lll}\hat{E}_{1}^{T} & \hat{A}_{2}^{T} & \Delta_{-\tau} \widetilde{B}_{3}^{T}\end{array}\right]$ is of square size.

iii) Suppose that an initial function $\phi$ is $\mu$-times continuously differentiable on $[-\tau, 0]$. Then it is consistent if and only if

$$
\begin{aligned}
\hat{A}_{2}(0) \phi(0)+\sum_{i=0}^{\mu} \hat{B}_{2, i}(0) \phi^{(i)}(-\tau)+\hat{f}_{2}(0) & =0, \\
\Delta_{-\tau} \widetilde{B}_{3} \phi+\Delta_{-\tau} \tilde{f}_{3} & =0, \text { for all } t \in[-\tau, 0] .
\end{aligned}
$$

The next corollary addresses the smoothness requirements of the initial function $\phi$ and the inhomogeneity $f$, for the existence of a solution $x$, which can be easily obtained from (4.14).

Corollary 4.8. Consider the DDAE (1.1) and its reformulation 4.10). Moreover, suppose that the sets $\mathbb{M}_{0}$ and $\widetilde{\mathbb{M}}_{0}$ defined by (4.13) coincide. Then, the following assertions hold.

i) Let $\tilde{\mu}$ be the largest index $i, 1 \leqslant i \leqslant \mu$, that $\hat{B}_{2, i}$, is not identical zero. Then, for any $k \in \mathbb{N}_{0}$, to make sure that the function $\left.x\right|_{[k \tau,(k+1) \tau]}$ is continuous, the function $\left.x\right|_{[(k-1) \tau, k \tau]}$ must be $\tilde{\mu}$-times continuously differentiable.

ii) To guarantee that the solution $x$ exists and it is at least continuous on the time interval $[-\tau, k \tau]$, an initial function $\phi$ has to be $k \cdot \tilde{\mu}$ times continuously differentiable.

iii) Suppose that the initial function $\phi$ is sufficiently smooth, then in order to guarantee that $x$ is continuous at every point $t \in \mathbb{I}$, the function $f$ has to be $\mu$-times continuously differentiable.

In Theorem 4.6 and Corollaries 4.7, 4.8 we have studied the structural properties of the DDAE (1.1) in the case $\mathbb{M}_{0}=\widetilde{\mathbb{M}}_{0}$. We now consider the general case, $\widetilde{\mathbb{M}}_{0} \subsetneq \mathbb{M}_{0}$. In the general case, one 
may introduce new variables to transform the high-order system 4.10 into first order form (1.1) as

$$
\begin{aligned}
& {\left[\begin{array}{ccccc}
\hat{E}_{1} & 0 & \ldots & 0 & 0 \\
\hline I_{n} & 0 & & & \\
& \ddots & \ddots & & \\
& & \ddots & 0 & \\
& & & I_{n} & 0 \\
\hline 0 & 0 & \ldots & 0 & 0 \\
\hline 0 & 0 & \ldots & 0 & 0 \\
0 & 0 & \ldots & 0 & 0 \\
0 & 0 & \ldots & 0 & 0
\end{array}\right]\left[\begin{array}{c}
\dot{x} \\
\ddot{x} \\
\vdots \\
x^{(\mu)} \\
x^{(\mu+1)}
\end{array}\right]=\left[\begin{array}{ccccc}
\hat{A}_{1} & 0 & \ldots & 0 & 0 \\
& I_{n} & & & \\
& & \ddots & & \\
& & & I_{n} & \\
& & & & I_{n} \\
\hat{A}_{2} & 0 & \ldots & 0 & 0 \\
0 & 0 & \ldots & 0 & 0 \\
0 & 0 & \ldots & 0 & 0 \\
0 & 0 & \ldots & 0 & 0
\end{array}\right] \underbrace{\left[\begin{array}{c}
x \\
\dot{x} \\
\vdots \\
x^{(\mu-1)} \\
x^{(\mu)}
\end{array}\right]}_{\chi}+}
\end{aligned}
$$

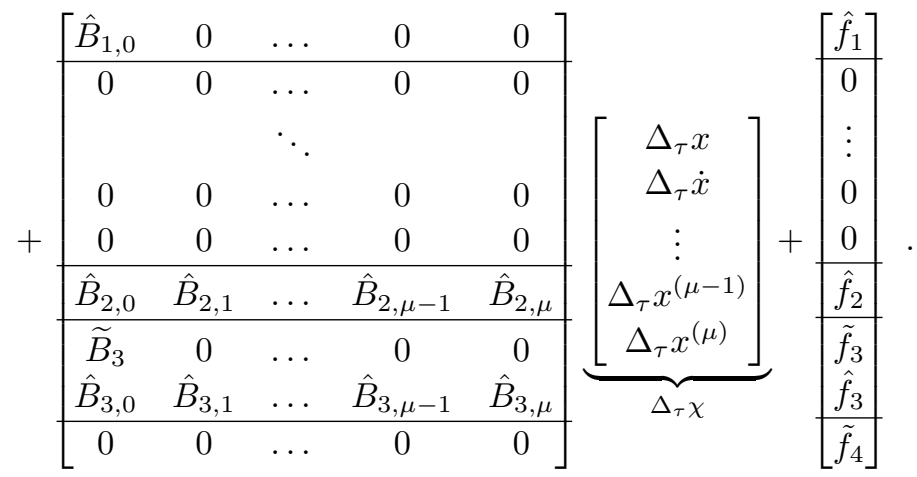

The advantage of this order reduction is that all the differential-algebraic equations for $\Delta_{\tau} x$ become algebraic constraints for $\Delta_{\tau} \chi$. However, as has been pointed out in [23, since it is not necessary to differentiate all components of $x$ really $\mu$ times, one should relax the order reduction (4.15) as follows.

Let $x=\left[\begin{array}{lll}x_{1} & \ldots & x_{n}\end{array}\right]^{T} \in \mathbb{C}^{n}$, and for $i=1, \ldots, n$, let $\rho_{i}$ be the highest order of derivatives of $x_{i}(t-\tau)$ occurring in 4.10 , we define

$$
X:=\xi(x), \xi: x \mapsto\left[\begin{array}{lllllll}
x_{1} & \ldots & x_{1}^{\left(\rho_{1}\right)} & \ldots & x_{n} & \ldots & x_{n}^{\left(\rho_{n}\right)}
\end{array}\right]^{T} .
$$

By introducing the variable $X$, and the corresponding relation

$$
{\dot{x_{i}}}^{(j-1)}=x_{i}^{(j)}, i=1, \ldots, n, j=1, \ldots, \rho_{i},
$$

we do not make any extra smoothness assumption and obtain a reduced-order system which will be denoted by

$$
\mathcal{E}^{1} \dot{X}=\mathcal{A}^{1} X+\mathcal{B}^{1} \Delta_{\tau} X+\mathcal{F}^{1}
$$

The function $x$ solves system 1.1 if and only if $X$ solves system (4.16). Furthermore, once knowing the value of $X$ at the point $t$, one also knows the value of $x(t)$.

Applying Procedures 4.2, 4.3 to system 4.16, we construct two sequences of sets $\left\{\mathbb{M}_{j}, j \in\right.$ $\left.\mathbb{N}_{0}\right\},\left\{\widetilde{\mathbb{M}}_{j}, j \in \mathbb{N}_{0}\right\}$ by the following procedure.

PROCEDURE 4.9.

Input: A DDAE of the form 1.1 
Begin: Set $j=0$ and let $\mathcal{E}^{0}:=E, \mathcal{A}^{0}:=A, \mathcal{B}^{0}=B, \mathcal{F}^{0}:=f, X^{0}:=\xi^{0}(x):=x$.

Step 1. Apply Procedure 4.2 to the equation

$$
\mathcal{E}^{j} \dot{X}^{j}=\mathcal{A}^{j} X^{j}+\mathcal{B}^{j} \Delta_{\tau} X^{j}+\mathcal{F}^{j}
$$

to obtain

$$
\left[\begin{array}{c}
\mathcal{E}_{1} \\
0 \\
0 \\
0
\end{array}\right] \dot{X}^{j}=\left[\begin{array}{c}
\mathcal{A}_{1} \\
\widetilde{\mathcal{A}}_{2} \\
0 \\
0
\end{array}\right] X^{j}+\left[\begin{array}{c}
\mathcal{B}_{1} \\
\widetilde{\mathcal{B}}_{2} \\
\widetilde{\mathcal{B}}_{3} \\
0
\end{array}\right] \Delta_{\tau} X^{j}+\left[\begin{array}{c}
\mathcal{F}_{1} \\
\widetilde{\mathcal{F}}_{2} \\
\widetilde{\mathcal{F}}_{3} \\
\widetilde{\mathcal{F}}_{4}
\end{array}\right]
$$

where the functions $\mathcal{E}_{1},\left[\begin{array}{c}\widetilde{\mathcal{A}}_{2} \\ \Delta_{-\tau} \widetilde{\mathcal{B}}_{3}\end{array}\right]$ have pointwise full row rank, and the pair $\left(\widetilde{\mathcal{B}}_{2}, \widetilde{\mathcal{B}}_{3}\right)$ has no hidden redundancy.

Step 2. Apply Procedure 4.3 to system 4.17 to obtain

$$
\left[\begin{array}{c}
\widehat{\mathcal{E}}_{1} \\
0 \\
0 \\
0 \\
0
\end{array}\right] \dot{X}^{j}=\left[\begin{array}{c}
\widehat{\mathcal{A}}_{1} \\
\widehat{\mathcal{A}}_{2} \\
0 \\
0 \\
0
\end{array}\right] X^{j}+\left[\begin{array}{c}
\widehat{\mathcal{B}}_{1,0} \\
\widehat{\mathcal{B}}_{2,0} \\
\widetilde{\mathcal{B}}_{3} \\
\widehat{\mathcal{B}}_{3,0} \\
0
\end{array}\right] \Delta_{\tau} X^{j}+\sum_{i=1}^{\mu_{j}}\left[\begin{array}{c}
0 \\
\widehat{\mathcal{B}}_{2, i} \\
0 \\
\widehat{\mathcal{B}}_{3, i} \\
0
\end{array}\right] \Delta_{\tau}\left(X^{j}\right)^{(i)}+\left[\begin{array}{c}
\widehat{\mathcal{F}}_{1} \\
\widehat{\mathcal{F}}_{2} \\
\widetilde{\mathcal{F}}_{3} \\
\widehat{\mathcal{F}}_{3} \\
\widetilde{\mathcal{F}}_{4}
\end{array}\right],
$$

where the function $\left[\begin{array}{lll}\widehat{\mathcal{E}}_{1}^{T} & \widehat{\mathcal{A}}_{2}^{T} & \Delta_{-\tau} \widetilde{\mathcal{B}}_{3}^{T}\end{array}\right]^{T}$ has pointwise full row rank.

Let

$$
\begin{aligned}
& \mathbb{M}_{j}:=\left\{x: \mathbb{I}_{\tau} \rightarrow \mathbb{C}^{n} \mid \widetilde{\mathcal{B}}_{3} \Delta_{\tau} X^{j}+\widetilde{\mathcal{F}}_{3}=0, t \in \mathbb{I}\right\} \\
& \widetilde{\mathbb{M}}_{j}:=\left\{x \in \mathbb{M}_{j} \mid \sum_{i=0}^{\mu_{j}} \widehat{\mathcal{B}}_{3, i} \Delta_{\tau}\left(X^{j}\right)^{(i)}+\widehat{\mathcal{F}}_{3}=0, t \in \mathbb{I}\right\},
\end{aligned}
$$

where $X^{j}=\left(\xi^{j-1} \circ \cdots \circ \xi^{0}\right)(x): \mathbb{I}_{\tau} \rightarrow \mathbb{C}^{\eta_{j}}$ with $\eta_{j}$ is the length of the vector-valued function $X^{j}$. If $\mathbb{M}_{j}=\widetilde{\mathbb{M}}_{j}$ then $\boldsymbol{S T O P}$ with the resulting system 4.18,

else proceed to Step 3.

Step 3. Let $\rho_{i}$ be the highest order of derivatives of $\left(\Delta_{\tau} X^{j}\right)_{i}$ (the $i$-th component of $\left.\Delta_{\tau} X^{j}\right)$, $i=1, \ldots, \eta_{j}$. Introduce a new variable

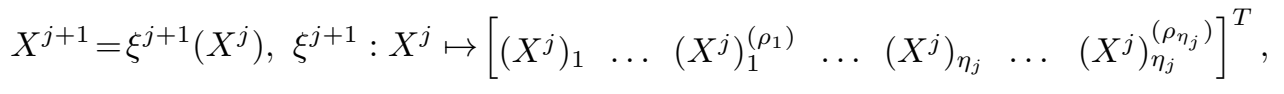

and rewrite system 4.18 as the first-order DDAE in $X^{j+1}$ according to 4.16

$$
\mathcal{E}^{j+1} \dot{X}^{j+1}=\mathcal{A}^{j+1} X^{j+1}+\mathcal{B}^{j+1} \Delta_{\tau} X^{j+1}+\mathcal{F}^{j+1},
$$

and repeat the process from Step 1.

End.

To show that Procedure 4.9 terminates after a finite number of steps, we observe that as discussed for system (4.15), by the order reduction, all the differential-algebraic equations for 
$\Delta_{-\tau} X^{j}$ will become algebraic equations for $\Delta_{-\tau} X^{j+1}$. Therefore, $\widetilde{\mathbb{M}}_{j} \supseteq \mathbb{M}_{j+1}$ for every $j \in \mathbb{N}_{0}$ which leads to the relation

$$
\mathbb{M}_{0} \supseteq \widetilde{\mathbb{M}}_{0} \supseteq \cdots \supseteq \mathbb{M}_{j} \supseteq \widetilde{\mathbb{M}}_{j} \supseteq \mathbb{M}_{j+1} \supseteq \widetilde{\mathbb{M}}_{j+1} \supseteq \ldots
$$

Since the sequence $\left\{\mathbb{M}_{j} \mid j \in \mathbb{N}_{0}\right\}$ is decreasing, it follows that there exists some $j \in \mathbb{N}_{0}$ such that $\mathbb{M}_{j}=\mathbb{M}_{j+1}$ and. therefore, the relation $\mathbb{M}_{j} \supseteq \widetilde{\mathbb{M}}_{j} \supseteq \mathbb{M}_{j+1}$ implies that $\mathbb{M}_{j}=\widetilde{\mathbb{M}}_{j}$. So Procedure 4.9 terminates after finitely many iterations.

Let $\omega:=\min \left\{j \in \mathbb{N}_{0} \mid \mathbb{M}_{j}=\widetilde{\mathbb{M}}_{j}\right\}$, then the final system obtained by applying Procedure 4.9 to the DDAE (1.1) is system 4.18 for $j=\omega$ which we denote by

$$
\left[\begin{array}{c}
\breve{\mathcal{E}}_{1} \\
0 \\
0 \\
0 \\
0
\end{array}\right] \dot{X}^{\omega}=\left[\begin{array}{c}
\breve{\mathcal{A}}_{1} \\
\breve{\mathcal{A}}_{2} \\
0 \\
0 \\
0
\end{array}\right] X^{\omega}+\left[\begin{array}{c}
\breve{\mathcal{B}}_{1,0} \\
\breve{\mathcal{B}}_{2,0} \\
\breve{\mathcal{B}}_{3} \\
\breve{\mathcal{B}}_{3,0} \\
0
\end{array}\right] \Delta_{\tau} X^{\omega}+\sum_{i=1}^{\mu_{\omega}}\left[\begin{array}{c}
0 \\
\breve{\mathcal{B}}_{2, i} \\
0 \\
\breve{\mathcal{B}}_{3, i} \\
0
\end{array}\right] \Delta_{\tau}\left(X^{\omega}\right)^{(i)}+\left[\begin{array}{c}
\breve{\mathcal{F}}_{1} \\
\breve{\mathcal{F}}_{2} \\
\breve{\mathcal{F}}_{3} \\
\breve{\mathcal{H}}_{3} \\
\breve{\mathcal{F}}_{4}
\end{array}\right]
$$

where the fourth block row equation is redundant to the third block row equation due to $\mathbb{M}_{\omega}=\widetilde{\mathbb{M}}_{\omega}$, and therefore can be omitted as in 4.14). Moreover, the matrix-valued function $\left[\begin{array}{ccc}\breve{\mathcal{E}}_{1}^{T} & \breve{\mathcal{A}}_{2}^{T} & \Delta_{-\tau} \breve{\mathcal{B}}_{3}^{T}\end{array}\right]^{T}$ has pointwise full row rank.

We summarize our results above in the following theorem, which characterizes the solvability analysis of the DDAE (1.1).

Theorem 4.10. Consider the DDAE (1.1) and assume that Procedure 4.9 proceeds until termination with the resulting system

$$
\left[\begin{array}{c}
\breve{\mathcal{E}}_{1} \\
0 \\
0 \\
0
\end{array}\right] \dot{X}^{\omega}=\left[\begin{array}{c}
\breve{\mathcal{A}}_{1} \\
\breve{\mathcal{A}}_{2} \\
0 \\
0
\end{array}\right] X^{\omega}+\left[\begin{array}{c}
\breve{\mathcal{B}}_{1,0} \\
\breve{\mathcal{B}}_{2,0} \\
\breve{\mathcal{B}}_{3} \\
0
\end{array}\right] \Delta_{\tau} X^{\omega}+\sum_{i=1}^{\mu_{\omega}}\left[\begin{array}{c}
0 \\
\breve{\mathcal{B}}_{2, i} \\
0 \\
0
\end{array}\right] \Delta_{\tau}\left(X^{\omega}\right)^{(i)}+\left[\begin{array}{c}
\breve{\mathcal{F}}_{1} \\
\breve{\mathcal{F}}_{2} \\
\breve{\mathcal{F}}_{3} \\
\breve{\mathcal{F}}_{4}
\end{array}\right]
$$

where the matrix-valued function $\left[\begin{array}{cccc}\breve{\mathcal{E}}_{1}^{T} & \breve{\mathcal{A}}_{2}^{T} & \Delta_{-\tau} \breve{\mathcal{B}}_{3}^{T}\end{array}\right]^{T}$ has pointwise full row rank. Then the DDAE (1.1) is solvable (resp. regular) if and only if system 4.20 has a solution (resp., has a unique solution). Precisely, provided that an initial function $\phi$ is sufficiently smooth, then system (1.1) is solvable if and only if

$$
\breve{\mathcal{F}}_{4}=0
$$

and it is regular if in addition the matrix-valued function $\left[\begin{array}{cccc}\breve{\mathcal{E}}_{1}^{T} & \breve{\mathcal{A}}_{2}^{T} & \Delta_{-\tau} \breve{\mathcal{B}}_{3}^{T}\end{array}\right]$ has as many rows as columns. Furthermore, components of $x(t)$ are also elements of $X^{\omega}(t)$, so by solving system 4.20, we also obtain the solution $x(t)$ of system (1.1.

Proof. By interchanging the roles of $x$ and $X^{\omega}$, the proof can be obtained by using the same argument as in the proof of Theorem 4.6 .

REMARK 4.11. The fact that $\mathbb{M}_{\omega}=\widetilde{\mathbb{M}}_{\omega}$ brings us back to the same situation as the case $\mathbb{M}_{0}=\widetilde{\mathbb{M}}_{0}$, and we obtain similar results as Corollaries 4.7, 4.8 by interchanging the roles of $x$ and $X^{\omega}$. Consequently, the structural properties, i.e., consistency and smoothness requirements for initial functions and inhomogeneities of 1.1 will follow. Moreover, for the numerical analysis it is useful to shift the third block row equation of system 4.20 before applying a numerical method. 

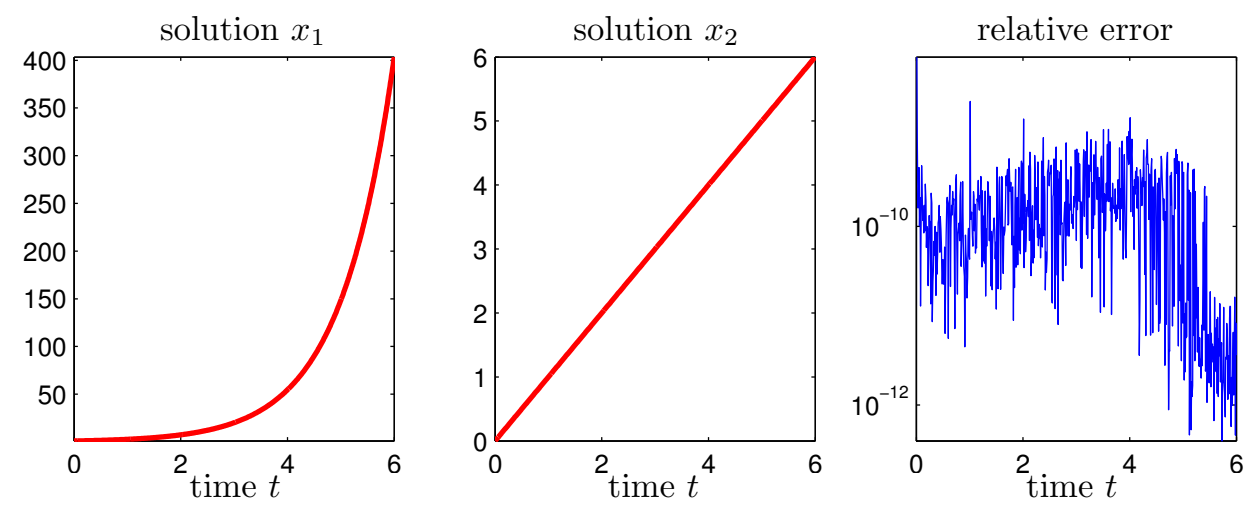

FIG. 5.1. Solution of system 5.2 together with the relative error $\left\|x(t)-x_{e}(t)\right\|_{\infty} /\left\|x_{e}(t)\right\|_{\infty}$

5. Numerical Examples. We illustrate our results by considering the numerical solution of two DDAEs of the form (1.1). As integration scheme we use a 3 stage Radau IIA method, which has been successfully implemented in the solver RADAR5 [15]. In the following two examples, we will show that the Radau IIA method may fail to handle DDAEs of the form (1.1). However, this difficulty can be overcome by using Radau IIA method for the resulting system obtained by applying Procedure 4.9 to 1.1 .

EXAmple 5.1. Consider the DDAE

$$
\left[\begin{array}{ll}
1 & t \\
0 & 0
\end{array}\right]\left[\begin{array}{l}
\dot{x}_{1}(t) \\
\dot{x}_{2}(t)
\end{array}\right]=\left[\begin{array}{cc}
0 & 1 \\
1 & t-1
\end{array}\right]\left[\begin{array}{l}
x_{1}(t-1) \\
x_{2}(t-1)
\end{array}\right]+\left[\begin{array}{c}
e^{t}+1 \\
-e^{t-1}-(t-1)^{2}
\end{array}\right]
$$

on the time interval $t \in \mathbb{I}=[0, \infty)$. Note that the associated DAE (4.1) of system (5.1) is underdetermined, and it is not suitable for numerical integration.

Procedure 4.9 applied to (5.1) will terminate after one iteration, due to the equality

$$
\widetilde{\mathbb{M}}_{0}=\mathbb{M}_{0}=\left\{x:[-1, \infty) \rightarrow \mathbb{C}^{2} \mid x_{1}(t-1)+(t-1) x_{2}(t-1)-e^{t-1}-(t-1)^{2}=0\right\} .
$$

The reformulated system is

$$
\left[\begin{array}{l}
0 \\
0
\end{array}\right]=\left[\begin{array}{ll}
0 & 1 \\
0 & 0
\end{array}\right]\left[\begin{array}{l}
x_{1}(t) \\
x_{2}(t)
\end{array}\right]+\left[\begin{array}{cc}
0 & 1 \\
1 & t-1
\end{array}\right]\left[\begin{array}{l}
x_{1}(t-1) \\
x_{2}(t-1)
\end{array}\right]+\left[\begin{array}{c}
1-2 t \\
-e^{t-1}-(t-1)^{2}
\end{array}\right], t \in \mathbb{I} .
$$

Now let us consider the numerical solution of system (5.1) with a given initial function $\phi(t)=\left[\begin{array}{c}e^{t} \\ t\end{array}\right]$. To do that we shift the second equation of 5.2 to obtain

$$
\left[\begin{array}{l}
0 \\
0
\end{array}\right]=\left[\begin{array}{ll}
0 & 1 \\
1 & t
\end{array}\right]\left[\begin{array}{l}
x_{1}(t) \\
x_{2}(t)
\end{array}\right]+\left[\begin{array}{ll}
0 & 1 \\
0 & 0
\end{array}\right]\left[\begin{array}{l}
x_{1}(t-1) \\
x_{2}(t-1)
\end{array}\right]+\left[\begin{array}{c}
1-2 t \\
-e^{t}-t^{2}
\end{array}\right], t \in \mathbb{I}
$$

System 5.3 is not only regular, but its associated DAE 4.1 has differentiation index 1. Here we follow the index concept of [5]. The Radau IIA method fails to solve system (5.1), however, it successfully treated system (5.3), as shown in Figure 5.1. There $x_{e}$ is the exact solution, and the time step is $h=0.01$.

ExAmple 5.2. Consider the DDAE

$$
\left[\begin{array}{lll}
0 & 0 & 1 \\
0 & 0 & 0 \\
0 & 1 & 0
\end{array}\right]\left[\begin{array}{l}
\dot{x}_{1}(t) \\
\dot{x}_{2}(t) \\
\dot{x}_{3}(t)
\end{array}\right]=\left[\begin{array}{lll}
0 & 1 & 0 \\
0 & 0 & 1 \\
0 & 0 & 0
\end{array}\right]\left[\begin{array}{l}
x_{1}(t) \\
x_{2}(t) \\
x_{3}(t)
\end{array}\right]+\left[\begin{array}{lll}
0 & 0 & 0 \\
1 & 0 & 0 \\
0 & 0 & 0
\end{array}\right]\left[\begin{array}{l}
x_{1}(t-1) \\
x_{2}(t-1) \\
x_{3}(t-1)
\end{array}\right]+\left[\begin{array}{c}
-t \\
-1-e^{t-1} \\
1
\end{array}\right],
$$


on the time interval $\mathbb{I}=[0, \infty)$. Again, we note that the associated DAE (4.1) of system (5.4) is under-determined, and it is not suitable for integration.

Applying Procedure 4.9, the resulting system from the first step is

$$
\begin{gathered}
{\left[\begin{array}{lll}
0 & 0 & 0 \\
0 & 0 & 0 \\
0 & 0 & 0
\end{array}\right]\left[\begin{array}{l}
\dot{x}_{1}(t) \\
\dot{x}_{2}(t) \\
\dot{x}_{3}(t)
\end{array}\right]=\left[\begin{array}{lll}
0 & 1 & 0 \\
0 & 0 & 1 \\
0 & 0 & 0
\end{array}\right]\left[\begin{array}{l}
x_{1}(t) \\
x_{2}(t) \\
x_{3}(t)
\end{array}\right]+\left[\begin{array}{lll}
0 & 0 & 0 \\
1 & 0 & 0 \\
0 & 0 & 0
\end{array}\right]\left[\begin{array}{l}
x_{1}(t-1) \\
x_{2}(t-1) \\
x_{3}(t-1)
\end{array}\right]} \\
+\left[\begin{array}{lll}
1 & 0 & 0 \\
0 & 0 & 0 \\
0 & 0 & 0
\end{array}\right]\left[\begin{array}{l}
\dot{x}_{1}(t-1) \\
\dot{x}_{2}(t-1) \\
\dot{x}_{3}(t-1)
\end{array}\right]+\left[\begin{array}{lll}
0 & 0 & 0 \\
0 & 0 & 0 \\
1 & 0 & 0
\end{array}\right]\left[\begin{array}{l}
\ddot{x}_{1}(t-1) \\
\ddot{x}_{2}(t-1) \\
\ddot{x}_{3}(t-1)
\end{array}\right]+\left[\begin{array}{c}
-t-e^{t-1} \\
-1-e^{t-1} \\
-e^{t-1}
\end{array}\right] .
\end{gathered}
$$

The corresponding sets are

$$
\begin{aligned}
& \mathbb{M}_{0}=\left\{x:[-1, \infty) \rightarrow \mathbb{C}^{3}\right\}, \\
& \widetilde{\mathbb{M}}_{0}=\left\{x:[-1, \infty) \rightarrow \mathbb{C}^{3} \mid \ddot{x}_{1}(t-1)-e^{t-1}=0\right\} .
\end{aligned}
$$

Since $\widetilde{\mathbb{M}}_{0} \subsetneq \mathbb{M}_{0}$, we introduce a new variable

$$
X^{1}(t):=\left[\begin{array}{lllll}
x_{1}(t) & \dot{x}_{1}(t) & \ddot{x}_{1}(t) & x_{2}(t) & x_{3}(t)
\end{array}\right]^{T},
$$

and rewrite system (5.5) as a first order DDAE

$$
\begin{aligned}
& \underbrace{\left[\begin{array}{lllll}
1 & 0 & & & \\
0 & 1 & & & \\
\hline & & 0 & & \\
& & & 0 & \\
\hline & & & 0
\end{array}\right]}_{\mathcal{E}^{1}}\left[\begin{array}{c}
\dot{x}_{1}(t) \\
\ddot{x}_{1}(t) \\
\dddot{x}_{1}(t) \\
\dot{x}_{2}(t) \\
\dot{x}_{3}(t)
\end{array}\right]=\underbrace{\left[\begin{array}{lllll}
0 & 1 & & & \\
0 & 0 & 1 & & \\
& & 0 & 1 & \\
& & & 0 & 1 \\
& & & & 0
\end{array}\right]}_{\mathcal{A}^{1}} \underbrace{\left[\begin{array}{l}
x_{1}(t) \\
\dot{x}_{1}(t) \\
\ddot{x}_{1}(t) \\
x_{2}(t) \\
x_{3}(t)
\end{array}\right]}_{X^{1}(t)} \\
& +\underbrace{\left[\begin{array}{lllll}
0 & 0 & & & \\
0 & 0 & & & \\
0 & 1 & 0 & 0 & 0 \\
1 & 0 & 0 & 0 & 0 \\
0 & 0 & 1 & 0 & 0
\end{array}\right]}_{\mathcal{B}^{1}}\left[\begin{array}{l}
x_{1}(t-1) \\
\dot{x}_{1}(t-1) \\
\ddot{x}_{1}(t-1) \\
x_{2}(t-1) \\
x_{3}(t-1)
\end{array}\right]+\underbrace{\left[\begin{array}{c}
0 \\
0 \\
-t-e^{t-1} \\
-1-e^{t-1} \\
-e^{t-1}
\end{array}\right]}_{\mathcal{F}^{1}} .
\end{aligned}
$$

The second iteration of Procedure 4.9 is applied to the system $\mathcal{E}^{1} \dot{X}^{1}(t)=\mathcal{A}^{1} X^{1}(t)+\mathcal{B}^{1} X^{1}(t-$ $1)+\mathcal{F}^{1}(t)$, and the resulting system is exactly system (5.6). The corresponding sets are

$$
\mathbb{M}_{1}=\widetilde{\mathbb{M}}_{1}=\left\{x:[-1, \infty) \rightarrow \mathbb{C}^{3} \mid \ddot{x}_{1}(t-1)-e^{t-1}=0\right\}
$$

and thus Procedure 4.9 terminates after two steps.

To solve system (5.1) numerically with a given initial function $\phi(t)=\left[\begin{array}{lll}e^{t} & t & 1\end{array}\right]^{T}$, one first shifts 
solution $x_{1}$


solution $x_{2}$
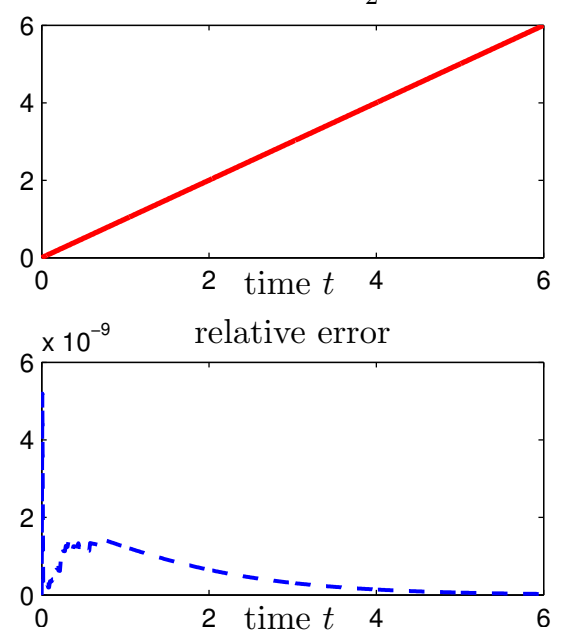

FIG. 5.2. Solution of system (5.4) obtained by solving 5.7 with 3-stage-Radau IIA method together with the relative error $\left\|x(t)-x_{e}(t)\right\|_{\infty} /\left\|x_{e}(t)\right\|_{\infty}$

forward the constraint of $x(t-1)$ in system (5.6), which is its last equation, to obtain

$$
\begin{aligned}
& {\left[\begin{array}{lllll}
1 & 0 & & & \\
0 & 1 & & & \\
\hline & & 0 & & \\
& & 0 & \\
\hline & & & 0
\end{array}\right]\left[\begin{array}{l}
\dot{x}_{1}(t) \\
\ddot{x}_{1}(t) \\
\dddot{x}_{1}(t) \\
\dot{x}_{2}(t) \\
\dot{x}_{3}(t)
\end{array}\right]=\left[\begin{array}{llll}
0 & 1 & & \\
0 & 0 & 1 & \\
& & 0 & 1 \\
& & 0 & 1 \\
& 1 & 0
\end{array}\right]\left[\begin{array}{l}
x_{1}(t) \\
\dot{x}_{1}(t) \\
\ddot{x}_{1}(t) \\
x_{2}(t) \\
x_{3}(t)
\end{array}\right]} \\
& +\left[\begin{array}{lllll}
0 & 0 & & \\
0 & 0 & & \\
0 & 1 & 0 & 0 & 0 \\
1 & 0 & 0 & 0 & 0 \\
0 & 0 & 0 & 0 & 0
\end{array}\right]\left[\begin{array}{l}
x_{1}(t-1) \\
\dot{x}_{1}(t-1) \\
\ddot{x}_{1}(t-1) \\
x_{2}(t-1) \\
x_{3}(t-1)
\end{array}\right]+\left[\begin{array}{c}
0 \\
0 \\
-t-e^{t-1} \\
-1-e^{t-1} \\
-e^{t}
\end{array}\right]
\end{aligned}
$$

The system (5.7) is not only regular, but its associated DAE (4.1) has differentiation index 1. Again, the Radau IIA method fails to solve system (5.4), but successfully solves system (5.7). The results are presented in Figure 5.2, and there $x_{e}$ is the exact solution, the time step is $h=0.01$.

6. Summary. The existence and uniqueness of solutions to general linear systems of delay differential-algebraic equations with variable coefficients has been studied. A reformulation procedure has been introduced to transform a given DDAE into one, where all the constraints, consistency conditions, smoothness requirements for the initial functions, and others solvability properties can be read off. It has been demonstrated that for some systems, without this reformulation step, numerical methods may fail or provide wrong results.

Acknowledgments. We thank Vinh Tho Ma for carrying the numerical simulations in Examples 5.1 and 5.2 .

\section{REFERENCES}

[1] U. Ascher and L. Petzold. Computer Methods for Ordinary Differential Equations and Differential-Algebraic Equations. SIAM Publications, 1998.

[2] U. M. Ascher and L. R. Petzold. The numerical solution of delay-differential algebraic equations of retarded and neutral type. SIAM J. Numer. Anal., 32:1635-1657, 1995.

[3] C. T. H. Baker, C. A. H. Paul, and H. Tian. Differential algebraic equations with after-effect. J. Comput. Appl. Math., 140(1-2):63-80, Mar. 2002. 
[4] A. Bellen and M. Zennaro. Numerical Methods for Delay Differential Equations. Oxford University Press, Oxford, UK, 2003.

[5] K. E. Brenan, S. L. Campbell, and L. R. Petzold. Numerical Solution of Initial-Value Problems in Differential Algebraic Equations. SIAM Publications, Philadelphia, PA, 2nd edition, 1996.

[6] S. L. Campbell. Singular linear systems of differential equations with delays. Appl. Anal., 2:129-136, 1980.

[7] S. L. Campbell. Nonregular 2D descriptor delay systems. IMA J. Math. Control Appl., 12:57-67, 1995.

[8] S. L. Campbell and V. H. Linh. Stability criteria for differential-algebraic equations with multiple delays and their numerical solutions. Appl. Math Comput., 208(2):397-415, 2009.

[9] T. Caraballo and J. Real. Navier-Stokes equations with delays. Proc. R. Soc. A, 457:2441-2453, 2001.

[10] L. Dieci and T. Eirola. On smooth decompositions of matrices. SIAM J. Matr. Anal. Appl., 20:800-819, 1999.

[11] M. J. Garrido-Atienza and P. Marín-Rubio. Navier-Stokes equations with delays on unbounded domains. Nonlinear Anal., 64(5):1100-1118, 2006.

[12] C. W. Gear. Simulation: conflicts between real-time and software. In Mathematical Software III, Proceedings of the Symposium at the University of Wisconsin, March 28-30, 1977, (ed. J.R. Rice), pages 121-138. Academic Press, New York, 1977.

[13] E. Griepentrog and R. März. Differential-Algebraic Equations and their Numerical Treatment. Teubner Verlag, Leipzig, Germany, 1986.

[14] C. Grossmann, H. Roos, and M. Stynes. Numerical Treatment of Partial Differential Equations. SpringerVerlag Berlin Heidelberg, 2007.

[15] N. Guglielmi and E. Hairer. Computing breaking points in implicit delay differential equations. Adv. Comput. Math., 29:229-247, 2008.

[16] P. Ha and V. Mehrmann. Analysis and reformulation of linear delay differential-algebraic equations. Electr. J. Lin. Alg., 23:703-730, 2012.

[17] E. Hairer and G. Wanner. Solving Ordinary Differential Equations II: Stiff and Differential-Algebraic Problems. Springer-Verlag, Berlin, Germany, 2nd edition, 1996.

[18] H. Heeb and A. Ruehli. Retarded models for PC board interconnects-or how the speed of light affects your spice circuit simulation. In IEEE International Conference on Computer-Aided Design, 1991. ICCAD-91, pages $70-73,1991$.

[19] C. Hollot, V. Misra, D. Towsley, and W. Gong. Analysis and design of controllers for AQM routers supporting TCP flows. IEEE Trans. Automat. Control, 47(6):945-959, 2002.

[20] F. Kelly. Mathematical modelling of the internet. In Mathematics Unlimited-2001 and Beyond, B. Engquist and W. Schmid, eds., pages 685-702. Springer-Verlag, Berlin, 2001.

[21] P. Kunkel and V. Mehrmann. Differential-Algebraic Equations: Analysis and Numerical Solution. EMS Publishing House, Zürich, Switzerland, 2006.

[22] W. Liu. Asymptotic behavior of solutions of time-delayed Burgers' equation. Discrete. Cont. Dyn.-B, 2(1):47$56,2002$.

[23] V. Mehrmann and C. Shi. Transformation of high order linear differential-algebraic systems to first order. Numer. Alg., 42:281-307, 2006.

[24] V. Misra, W. B. Gong, and D. Towsley. Fluid-based analysis of a network of AQM routers supporting TCP flows with an application to red. SIGCOMM Comput. Commun. Rev., 30(4):151-160, Aug. 2000.

[25] G. Planas and E. Hernndez. Asymptotic behaviour of two-dimensional time-delayed Navier-Stokes equations. Discrete. Contin. Dyn. S, 21(4):1245-1258, 2008.

[26] R. Riaza. Differential-algebraic systems: Analytical aspects and circuit applications. World Scientific Publishing Co. Pte. Ltd., Hackensack, NJ., 2008.

[27] L. F. Shampine and P. Gahinet. Delay-differential-algebraic equations in control theory. Appl. Numer. Math., 56(3-4):574-588, Mar. 2006.

[28] A. Steinbrecher. Regularization of quasi-linear differential-algebraic equations in multibody dynamics. In Proceedings of Multibody Dynamics 2011-ECCOMAS Thematic Conference Brussels (Bruxelles, Belgium, July 4-7, 2011), 2011.

[29] M. Wim and N. Silviu-Iulian. Stability and Stabilization of Time-Delay Systems: An Eigenvalue-based Approach. SIAM, 2007.

[30] Q. Zhong. Robust Control of Time-delay Systems. Springer, 2006. 